\title{
Temporary Protection of $\boldsymbol{H}$-Phosphinic Acids as a Synthetic Strategy
}

\author{
Laëtitia Coudray ${ }^{a}$ and Jean-Luc Montchampa \\ Jean-Luc Montchamp: j.montchamp@tcu.edu \\ aDepartment of Chemistry, Box 298860, Texas Christian University, Fort Worth, Texas 76129, \\ USA. Phone: 1+(817) 257 6201, Fax: 1+(817) 2575851
}

\section{Abstract}

$H$-Phosphinates obtained through various methodologies are protected directly via reaction with triethyl orthoacetate. The resulting products can be manipulated easily, and various synthetic reactions are presented. For example, application to the synthesis of aspartate transcarbamoylase (ATCase) or kynureninase inhibitors are illustrated.

Other reactions, such as Sharpless' asymmetric dihydroxylation, or Grubbs' olefin crossmetathesis are also demonstrated.

\section{Keywords \\ Phosphorus; Palladium; Metathesis; Cross-Coupling; Synthesis}

\section{Introduction}

Gallagher, and soon afterwards chemists at Ciba-Geigy (formerly) have introduced and developed an ingenious family of reagents in which one $\mathrm{P}-\mathrm{H}$ bond of hypophosphorous acid (HPA, $\mathrm{H}_{3} \mathrm{PO}_{2}, \mathbf{1}$ ) is masked temporarily and then re-introduced in a later step. ${ }^{[1]}$ This allows selective reactions and solves the problem of disubstitution of the two reactive $\mathrm{P}-\mathrm{H}$ bonds in HPA. We have been referring to these reagents as "Ciba-Geigy reagents" [ethyl 1,1(diethoxyethyl)- and 1,1-(diethoxymethyl)- $H$-phosphinates, $\mathrm{RC}(\mathrm{OEt})_{2} \mathrm{P}(\mathrm{O})(\mathrm{OEt}) \mathrm{H}, \mathrm{R}=\mathrm{Me}$, $\mathrm{H} ; \mathbf{2}$ and $\mathbf{3}$, respectively], in keeping with their historical origins. Reagents $\mathbf{2}$ and $\mathbf{3}$ have permitted the synthesis of many phosphinic acid derivatives through alkylation, and they have been used rather extensively. ${ }^{[2]}$ Reagent $\mathbf{2}$ is often superior to reagent 3 because cleavage of the acetal functionality takes place under milder conditions and without cleavage of the $\mathrm{P}(\mathrm{O}) \mathrm{OEt}$ ester group. ${ }^{[1 \mathrm{~d}]}$

(๔ WILEY-VCH Verlag GmbH \& Co. KGaA, 69451 Weinheim, Germany, 2009)

Correspondence to: Jean-Luc Montchamp, j.mont champ@tcu . edu.

Supporting information for this article is available on the WWW under http://www.eurjoc.org/.

Supporting Information (see footnote on the first page of this article): General chemistry (1 page); procedures and spectral data (18 pages); ${ }^{1} \mathrm{H},{ }^{13} \mathrm{C}$ and ${ }^{31} \mathrm{P}$ NMR spectra (135 pages). 
<smiles>CCOC(C)(OCC)P(=O)=O</smiles>

2

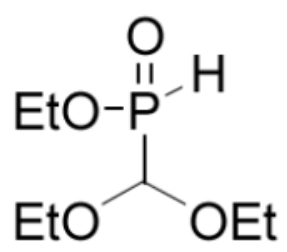

3

Over the past ten years, we have developed several reactions for the synthesis of $H$ phosphinates, directly from hypophosphorous precursors (HPA, anilinium and sodium hypophosphites, alkyl phosphinates, etc.). ${ }^{[3]}$ Many of these reactions provide an environmentally benign access to $H$-phosphinates, because halogenated compounds and/or stoichiometric strong bases are avoided, the reactions proceed with low loadings of transition-metal catalysts or with a radical initiator, and either no by-product is created (addition/hydrophosphinylation) or water is formed (condensation/cross-coupling). As a result, many $H$-phosphinates are now accessible directly, through routes unavailable to the Ciba-Geigy reagents. However, $H$-phosphinates are often not compatible with many reactions (oxidation/reduction, etc.) that would modify the carbon chain, so a temporary protection scheme is sometimes necessary. We recently reported one solution to this problem, based on borane complexes. ${ }^{[4]}$ Since our methodologies provide compounds which cannot be made easily (or efficiently) from reagents $\mathbf{2}$ or $\mathbf{3}$, we decided to investigate a protection strategy based on acetals. Perhaps surprisingly, few reports have described the reaction of $H$-phosphinates with orthoesters. There is only one reference that describes the reaction of a single $H$-phosphinic acid with triethyl orthoformate, ${ }^{[5]}$ and none with triethyl orthoacetate (Table 1).

During the synthesis of an aspartate transcarbamoylase (ATCase) inhibitor, we recently were confronted with the need to protect cinnamyl- $H$-phosphinic acid before an ozonolysis step, and we successfully employed triethyl orthoacetate to prepare $\mathbf{4}$ in excellent yield (Eq 1). ${ }^{[11]}$ We realized that this strategy would be synthetically useful, and this prompted the present study on the direct synthesis of various ethyl (1,1-diethoxyethyl)phosphinates from $H$-phosphinic acids, and some of their reactivity.

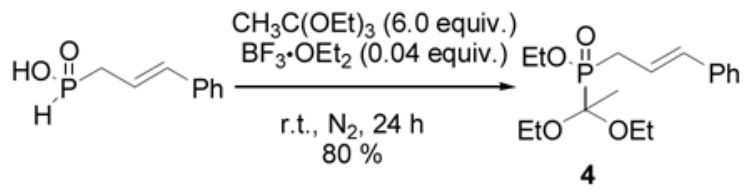

Herein, we report on the reaction of $H$-phosphinic acids with triethyl orthoacetate, as well as some subsequent transformations not previously exploited in the literature. The synthetic transformations illustrated include oxidations (ozonolysis, epoxidation, Sharpless' asymmetric dihydroxylation), reductions (hydrogenation, hydroboration), BuchwaldHartwig amination, and olefin cross-metathesis. All these reactions are not compatible with free $\mathrm{P}-\mathrm{H}$ bonds, at least in our hands. 


\section{Results and Discussion}

The advantages of our strategy (Scheme 1), over the use of the Ciba-Geigy reagents, stems from the much wider availability of $H$-phosphinates $\mathbf{5}$ using our methodologies, as well as the more practical nature of using triethyl orthoacetate on $H$-phosphinates 5 instead of HPA $\mathbf{1}$, or the simpler purification of the products 5 as opposed to purifying $\mathbf{2}$ and $\mathbf{3}$. Thus, although the two strategies are only differing in the order of the steps, the limited transformations available from $\mathbf{2}$ (or $\mathbf{3}$ ) to compounds $\mathbf{6}$ are narrowing the scope of these reagents. For example, $\mathbf{2}$ and $\mathbf{3}$ can be alkylated using a strong base and an electrophile, they can be silylated and alkylated, and some cross-coupling chemistry has been reported (with high loadings of palladium catalyst).

Even our protecting strategy based on phosphonite-borane complexes $\mathbf{7}$ obtained either from 5 or 9 (Scheme 1), ${ }^{[4]}$ is not competitive since, for example, the simple hydrogenation of an alkene is sluggish (Eq 2), and if the same reaction is conducted in methanol, decomplexation of the borane takes place exclusively.

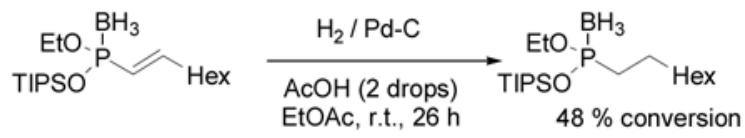

The protection of dichlorophosphines $\mathbf{8}$ (Scheme 1 and Table 1) has been used, especially with $\mathrm{ClCH}_{2} \mathrm{PCl}_{2}$, but the lack of availability of these compounds is a significant limitation (in fact, $\mathbf{8}$ can be made from $\mathbf{5}, \mathrm{R}^{2}=\mathrm{H}$ ).

\section{Synthesis of phosphinate acetals 6 from $H$-phosphinates 5}

Since the reaction of $H$-phosphinic acids with triethyl orthoacetate has not been described previously ${ }^{[12]}$ we decided to investigate its scope. Table 2 shows the results of this study. All the substrates are readily available through various methodologies we have developed. Isolated yields of protected acetals range from moderate to good (41-80\%) for a wide range of functionalized substrates.

As described for other phosphinylidene-containing compounds, $\mathrm{BF}_{3} \cdot \mathrm{Et}_{2} \mathrm{O}$ in a substoichiometric amount was satisfactory in general (Method A, Table 1). In some cases, a small amount of ytterbium triflate also gave good results, especially in hydroxy-containing substrates. In this case, some acetate is directly obtained as a mixture with the alcohol, so acetylation was conducted on the crude reaction mixture. With alcohol-containing substrates, partial esterification was observed, and acetylation was conducted to drive the reaction to completion (Method $\mathrm{E}$ ). With $\mathrm{BF}_{3}{ }^{\bullet} \mathrm{Et}_{2} \mathrm{O}$, a full equivalent was used for alcohols (Method D).

Triethyl orthoformate is less satisfactory (Method B), but only useful in cases where the $\mathrm{H}$ phosphinic acid intermediate is ultimately desired because selective deprotection is problematic. ${ }^{[2]}$ Attempts at using the literature condition $(p-\mathrm{TsOH})^{[5]}$, or $^{\mathrm{BF}_{3}} \mathrm{Et}_{2} \mathrm{O}$, did not give good results

\section{Synthetic applications with compounds 6}

Several reactions were tried on protected ethyl $H$-phosphinates 6 (Schemes 2 and 3). 
Dihydroxylation of allyl- $H$-phosphinate 10 (Scheme 2) proceeds in good yield to afford diol 11. Compound 11 can be used to prepare phospholipid analogs, and this will be investigated in future work. Hydrolysis of $\mathbf{1 1}$ afforded $H$-phosphinate $\mathbf{1 2}$ in quantitative yield. Diol 12 was also oxidized using our catalytic process ${ }^{[3 \mathrm{~h}]}$ to afford phosphonic acid $\mathbf{1 3}$.

Hydroboration/oxidation of $\mathbf{1 0}$ proceeded uneventfully to afford alcohol $\mathbf{1 4}$ in $71 \%$ yield. Another important reaction (Scheme 2) is the palladium-catalyzed cross-coupling of chloride 15. Buchwald-Hartwig amination took place in moderate yield to afford aniline derivative 16. Since $H$-phosphinates can undergo oxidation or cross-coupling, protection of the $\mathrm{P}-\mathrm{H}$ functionality is necessary to perform these reactions.

A series of reactions was performed on ethyl cinnamyl-1,1-(diethoxy)ethyl-phosphinate 4 (Scheme 3). Unlike the sluggish hydrogenation of borane complexes (Eq 2), hydrogenation of 4 under standard conditions takes place in quantitative yield to produce 17. The presence of a phosphinylidene functionality $\mathrm{P}(\mathrm{O}) \mathrm{H}$ seems to be incompatible with hydrogenation as we have never been able to conduct the heterogeneous hydrogenation of $H$-phosphinic acids or esters, most likely because of complexation to $\mathrm{Pd}$ and poisoning of the catalyst.

Hydroboration of $\mathbf{4}$ gave a mixture of regioisomers 18 (97\% NMR yield) in low yield after chromatographic separation of each isomer (18a and 18b). On the other hand, epoxidation gave racemic 19 in moderate yield. Finally, Sharpless' asymmetric dihydroxylation ${ }^{[13]}$ gave the corresponding diol 20 in good yield. Interestingly, no kinetic resolution was observed in the formation of 20. As in Scheme 2, acidic hydrolysis delivered the diol $\mathbf{2 1}$ in quantitative yield and $59 \%$ ee. The absolute configuration was assigned based on the Sharpless-Corey models. ${ }^{[14]}$ Enantiomeric excess determination was based on the formation of a diastereomeric salt with quinine and ${ }^{31} \mathrm{P}-\mathrm{NMR}$ analysis.

Another illustration of our strategy is an improved synthesis of a potent ATCase inhibitor $\left(\mathrm{K}_{\mathrm{i}}=0.42 \mu \mathrm{M}, 27\right.$, Scheme 4$) .{ }^{[11]}$ Initially, we used the temporary protection strategy with cinnamyl- $H$-phosphinic acid (Eq 1 ), but since no purification was conducted, the benzoic acid side product required an adjustment in stoichiometry for the amidation step. Instead, we realized that isoprenylation would give volatile acetone after ozonolysis/reduction and so the reaction product would be cleaner and the subsequent yield higher. We have previously described the hydrophosphinylation of isoprene, ${ }^{[3 \mathrm{n}]}$ and low catalyst loading can be employed. In spite of this, selection of our reusable polystyrene-supported catalyst ${ }^{[31]}$ for the hydrophosphinylation of isoprene was superior, resulting in a quantitative yield of product 22 after 5 runs of nixantphos-polymer 23 recycling (Scheme 4).

Isoprenyl- $H$-phosphinic acid 22 was protected according to method A (Table 1, entry 3 ) to produce $\mathbf{2 4}$. The synthesis of $\mathbf{2 2}$ and subsequently $\mathbf{2 4}$ is much more efficient and environmentally friendly than the alternative alkylation of $\mathbf{2}$ with isoprenyl halides (either through deprotonation or silylation of 2 ) would be (Scheme 1). Ozonolysis and oxidation of $\mathbf{2 4}$ with sodium chlorite ${ }^{[15]}$ gave the corresponding carboxylic acid $\mathbf{2 5}$, which was used without intermediate isolation. Deprotection of the resulting amide $\mathbf{2 6}$ through hydrogenolysis of the benzyl groups, and acid hydrolysis of the acetal provided inhibitor 27 in good yield after ion-exchange chromatography.

Another application of building block 24, this time through an ozonolysis/Strecker sequence is shown in Scheme 5. Compound $\mathbf{2 8}$ could be hydrolyzed to the phosphino analog $\mathbf{2 9}$ of aspartic acid, ${ }^{[16]}$ or it could be $N$-protected to 30, $P$-deprotected selectively, then crosscoupled to an aryl halide to form $\mathbf{3 2}^{[17]}$ after deprotection of intermediate $31 .{ }^{[18]}$ This strategy could be applied to the combinatorial synthesis of other kynureninase inhibitors 32 by selecting other aryl halides for coupling. 
Kynureninase inhibitors have potential for the blockage of quinolinic acid biosynthesis. Quinolinic acid has been implicated in various neurological disorders, such as epilepsy, Huntington's disease, and AIDS-related dementia. ${ }^{[17]}$ Thus, our method offers a flexible entry to prepare various analogs of $\mathbf{3 3}$ which could have useful biological activities.

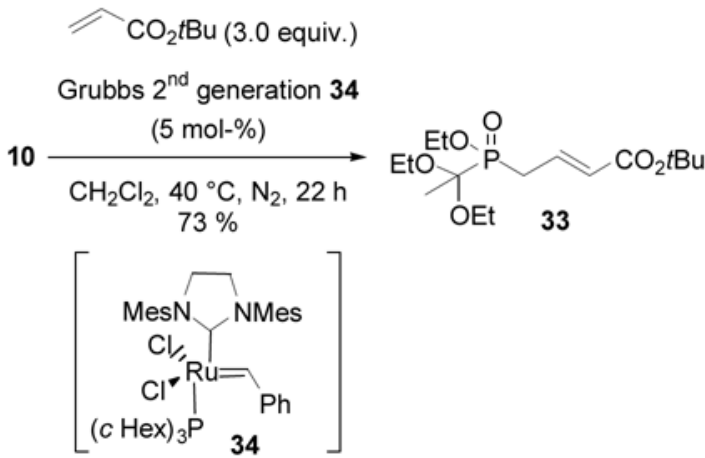

The last example of a reaction on protected phosphinic acid $\mathbf{1 0}$ is shown in Equation 3. Olefin metathesis of phosphorus-containing compounds, ${ }^{[19]}$ as well as cross-metathesis of phosphonates ${ }^{[20]}$ are known, but no example related to the cross-metathesis of phosphinic acids has ever been reported. Phosphinylidene containing compounds are not reactive partners in this reaction, but temporary protection clearly solves this problem. Crossmetathesis of $\mathbf{1 0}$ using the "second generation Grubbs' catalyst" 34 afforded acrylate $\mathbf{3 3}$ in good yield.

\section{Conclusions}

The temporary protection of $H$-phosphinic acids has not been described previously. In spite of 20 years of successful uses of the Ciba-Geigy reagents, a more efficient approach to useful synthetic intermediates has been developed. This manuscript describes the synthesis of various temporarily protected $H$-phosphinic acids for the synthesis of more complex intermediates. Since numerous $H$-phosphinic acids are now readily available using various environmentally benign methodologies we have developed, the present strategy offers an attractive alternative to others previously described in the literature. For certain $H$ phosphinic acids which can be difficult to purify, this method also allows purification and esterification, so the purified ethyl $H$-phosphinate ester can be obtained easily after treatment with $\mathrm{TMSCl} / \mathrm{CHCl}_{3}$. Even if P-diastereoselective reactions were not observed, and kinetic resolution could not be accomplished for the preparation of P-chiral compounds, the present approach should be useful to phosphorus chemists in general. From dihydroxylation, to cross-coupling, to cross-metathesis; the temporary protection of $H$-phosphinic acids as the corresponding acetals offers a new and efficient approach toward functional intermediates. Since deprotection of the 1,1-(diethoxy)ethyl group can deliver $H$-phosphinate esters or acids, a wide range of intermediates is accessible. Future work from our laboratory will investigate the synthesis of phospholipid analogs, and additional asymmetric reactions on protected phosphinates $\mathbf{6}$, as well as Tsuji-Trost type allylation reactions on unsaturated acetates. 


\section{Experimental Section}

\section{General procedure for the protection of $\boldsymbol{H}$-phosphinates (Table 2, Method A)}

To $H$-phosphinic acid or $H$-phosphinic acid ethyl ester (5.0 mmol, 1.0 equiv.) were added triethyl orthoacetate $(5.5 \mathrm{~mL}, 30.0 \mathrm{mmol}, 6.0$ equiv.) and boron trifluoride diethyl ether ( 0.13 or $0.31 \mathrm{~mL}, 1.0$ or $2.5 \mathrm{mmol}, 0.2$ or 0.5 equiv.) at r.t.. The reaction mixture was vigorously stirred for $16-24 \mathrm{~h}$ under $\mathrm{N}_{2}$ at r.t. to afford a yellow solution. The reaction mixture was diluted with ethyl acetate $(50 \mathrm{~mL})$ and washed with $0.5 \mathrm{M}$ aqueous $\mathrm{NaHCO}_{3}(1$ $\times 15 \mathrm{~mL})$ then brine $(2 \times 15 \mathrm{~mL})$. The organic layer was dried with $\mathrm{MgSO}_{4}$ and concentrated in vacuo. The resulting oil was purified by chromatography over silica to afford the clean product.

(1,1-Diethoxy-ethyl)-cinnamyl-phosphinic acid ethyl ester 4 (Equation 1, Table 2, Entries 1a and 2a)

Method A, 0.2 eq. $\mathrm{BF}_{3} \cdot \mathrm{OEt}_{2}, 24 \mathrm{~h}$, using cinnamyl- $H$-phosphinic acid. ${ }^{[21]}$ Chromatography over silica gel (hexanes / ethyl acetate, 6/4, v/v) afforded 4 as a light yellow oil $(1.3 \mathrm{~g}, 80 \%$ yield). ${ }^{1} \mathrm{H}$ NMR (300 MHz, $\left.\mathrm{CDCl}_{3}\right)$ \&: 7.20-7.38 (m, $5 \mathrm{H}$, aro $\left.\mathrm{CH}\right), 6.53\left(\mathrm{dd},{ }^{3} \mathrm{~J}_{\mathrm{H}, \mathrm{H}}=16.0\right.$ $\left.\mathrm{Hz},{ }^{4} \mathrm{~J}_{\mathrm{HCCC}}=4.0 \mathrm{~Hz}, 1 \mathrm{H},-\mathrm{CH}=\right), 6.19-6.31(\mathrm{~m}, 1 \mathrm{H},-\mathrm{CH}=), 4.15-4.28\left(\mathrm{~m}, 2 \mathrm{H},-\mathrm{CH}_{2}-\mathrm{O}\right)$, 3.60-3.83 (m, $\left.4 \mathrm{H},-\mathrm{CH}_{2}-\mathrm{O}\right), 2.71-2.92\left(\mathrm{~m}, 2 \mathrm{H},-\mathrm{CH}_{2}-\mathrm{P}\right), 1.54\left(\mathrm{~d},{ }^{3} J_{\mathrm{HCCP}}=11.0 \mathrm{~Hz}, 3 \mathrm{H}\right.$,

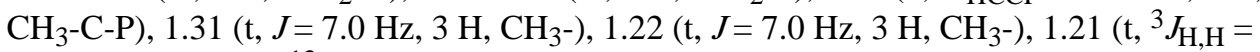
$\left.7.0 \mathrm{~Hz}, 3 \mathrm{H}, \mathrm{CH}_{3^{-}}\right) ;{ }^{13} \mathrm{C} \mathrm{NMR}\left(75.45 \mathrm{MHz}, \mathrm{CDCl}_{3}\right) \delta: 137.3,134.8\left(\mathrm{~d}, J_{\mathrm{PCCC}}=12.0 \mathrm{~Hz}\right)$, $128.8(2 \mathrm{C}), 127.7,126.4\left(\mathrm{~d}, J_{\mathrm{PCCCCC}}=1.5 \mathrm{~Hz}, 2 \mathrm{C}\right), 119.1\left(\mathrm{~d}, J_{\mathrm{PCC}}=10.5 \mathrm{~Hz}\right), 101.5(\mathrm{~d}$, $\left.J_{\mathrm{PC}}=139.0 \mathrm{~Hz}\right), 62.1\left(\mathrm{~d}, J_{\mathrm{POC}}=7.0 \mathrm{~Hz}\right), 58.5\left(\mathrm{~d}, J_{\mathrm{PCOC}}=5.0 \mathrm{~Hz}\right), 57.0\left(\mathrm{~d}, J_{\mathrm{PCOC}}=7.0\right.$ $\mathrm{Hz}), 31.7\left(\mathrm{~d}, J_{\mathrm{PC}}=82.5 \mathrm{~Hz}\right), 20.8\left(\mathrm{~d}, J_{\mathrm{PCC}}=12.0 \mathrm{~Hz}\right), 17.0\left(\mathrm{~d}, J_{\mathrm{POCC}}=5.0 \mathrm{~Hz}\right), 15.7$, $15.5 ;{ }^{31} \mathrm{P}$ NMR (121.47 MHz, $\mathrm{CDCl}_{3}$ ) $\delta: 45.96$ (s); HRMS (EI+) calcd. for $\mathrm{C}_{15} \mathrm{H}_{22} \mathrm{O}_{3} \mathrm{P}$, $\left([\mathrm{M}-\mathrm{OEt}]^{+}\right)$281.1307, found 281.1302 .

\section{(1,1-Diethoxy-ethyl)-(4-phenylamino-phenyl)-phosphinic acid ethyl ester 16 (Scheme 2)}

In a sealed tube, $\mathrm{K}_{3} \mathrm{PO}_{4}\left(0.297 \mathrm{~g}, 1.4 \mathrm{mmol}, 1.4\right.$ equiv.), $\mathrm{Pd}_{2} \mathrm{dba}_{3}(0.0092 \mathrm{~g}, 0.01 \mathrm{mmol}, 4.0$ mol-\%) and 2-dicyclohexylphosphino-2'-(N,N-dimethylamino)biphenyl (0.017 g, 0.044 mmol, $4.4 \mathrm{~mol}-\%)$ were added to the ester $15(0.32 \mathrm{~g}, 1.0 \mathrm{mmol})$ in DME $(2.0 \mathrm{~mL})$ at r.t.. After $24 \mathrm{~h}$ at $100^{\circ} \mathrm{C}$, the reaction mixture was cooled down and filtered through Celite ${ }^{\circledR}$. The solution was concentrated in vacuo and the resulting oil was purified by chromatography over silica gel (hexanes / ethyl acetate, 4/6, v/v) to afford the amine $\mathbf{1 6}$ as a colorless oil $(0.197 \mathrm{~g}, 52 \%) .{ }^{1} \mathrm{H}$ NMR $\left(300 \mathrm{MHz}, \mathrm{CDCl}_{3}\right) \delta: 7.70\left(\mathrm{dd},{ }^{3} \mathrm{~J}_{\mathrm{H}, \mathrm{H}}=10.5 \mathrm{~Hz},{ }^{3} J_{\mathrm{H}, \mathrm{H}}=8.5 \mathrm{~Hz}, 2\right.$ $\mathrm{H}$, aro $\mathrm{CH}), 7.30-7.36(\mathrm{~m}, 2 \mathrm{H}$, aro $\mathrm{CH}), 7.18\left(\mathrm{~d},{ }^{3} J_{\mathrm{H}, \mathrm{H}}=8.5 \mathrm{~Hz}, 2 \mathrm{H}\right.$, aro $\left.\mathrm{CH}\right), 7.02-7.08$ (m, $3 \mathrm{H}$, aro $\mathrm{CH}), 4.02-4.26\left(\mathrm{~m}, 2 \mathrm{H},-\mathrm{CH}_{2}-\mathrm{O}\right), 3.56-3.80\left(\mathrm{~m}, 4 \mathrm{H},-\mathrm{CH}_{2}-\mathrm{O}\right), 1.45$ $\left(\mathrm{d},{ }^{3} J_{\mathrm{HCCP}}=12.0 \mathrm{~Hz}, 3 \mathrm{H}, \mathrm{CH}_{3}-\mathrm{C}-\mathrm{P}\right), 1.34\left(\mathrm{t},{ }^{3} J_{\mathrm{H}, \mathrm{H}}=7.0 \mathrm{~Hz}, 3 \mathrm{H}, \mathrm{CH}_{3}-\right), 1.19\left(\mathrm{t},{ }^{3} J_{\mathrm{H}, \mathrm{H}}=\right.$ $\left.7.0 \mathrm{~Hz}, 3 \mathrm{H}, \mathrm{CH}_{3^{-}}\right), 1.18\left(\mathrm{t},{ }^{3} J_{\mathrm{H}, \mathrm{H}}=7.0 \mathrm{~Hz}, 3 \mathrm{H}, \mathrm{CH}_{3^{-}}\right) ;{ }^{13} \mathrm{C} \mathrm{NMR}\left(75.45 \mathrm{MHz}, \mathrm{CDCl}_{3}\right) \delta$ : $148.1\left(\mathrm{~d}, J_{\mathrm{PCCCC}}=2.5 \mathrm{~Hz}\right), 141.4,134.7\left(\mathrm{~d}, J_{\mathrm{PCC}}=10.5 \mathrm{~Hz}, 2 \mathrm{C}\right), 129.5(2 \mathrm{C}), 122.8,120.5$ $(2 \mathrm{C}), 118.1\left(\mathrm{~d}, J_{\mathrm{PC}}=128.5 \mathrm{~Hz}\right), 114.9\left(\mathrm{~d}, J_{\mathrm{PCCC}}=13.0 \mathrm{~Hz}, 2 \mathrm{C}\right), 101.6\left(\mathrm{~d}, J_{\mathrm{PC}}=153.5 \mathrm{~Hz}\right)$, $61.4\left(\mathrm{~d}, J_{\mathrm{POC}}=7.0 \mathrm{~Hz}\right), 58.4\left(\mathrm{~d}, J_{\mathrm{PCOC}}=5.5 \mathrm{~Hz}\right), 58.0\left(\mathrm{~d}, J_{\mathrm{PCOC}}=7.0 \mathrm{~Hz}\right), 20.5\left(\mathrm{~d}, J_{\mathrm{PCC}}=\right.$ $13.0 \mathrm{~Hz}), 16.9\left(\mathrm{~d}, J_{\mathrm{POCC}}=5.5 \mathrm{~Hz}\right), 15.8,15.6 ;{ }^{31} \mathrm{P} \mathrm{NMR}\left(121.47 \mathrm{MHz}, \mathrm{CDCl}_{3}\right) \delta: 36.74(\mathrm{~s})$; HRMS (EI+) calcd. for $\mathrm{C}_{20} \mathrm{H}_{28} \mathrm{NO}_{4} \mathrm{P},\left([\mathrm{M}]^{+}\right)$377.1756, found 377.1754.

\section{Isoprenyl-H-phosphinic acid 22[3n] (Scheme 4, Table 2, Phosphinate, Entry 3): Synthesis with the polymer catalyst}

In a sealed tube, isoprene $\left(0.5 \mathrm{~mL}, 5.0 \mathrm{mmol}, 1.0\right.$ equiv.) was added to concentrated $\mathrm{H}_{3} \mathrm{PO}_{2}$ (0.495 g, $7.5 \mathrm{mmol}, 1.5$ equiv.) in acetonitrile $(10.0 \mathrm{~mL}, 0.5 \mathrm{M})$, followed by $\mathrm{Pd}_{2} \mathrm{dba}_{3}(0.023$ g, $0.025 \mathrm{mmol}, 0.5 \mathrm{~mol}-\%)$ and polystyrene nixantphos catalyst $\mathbf{2 3}^{[31]}(0.06 \mathrm{mmol}, 1.2 \mathrm{~mol}-$ $\%)$. After the tube was sealed, the reaction was heated at $85^{\circ} \mathrm{C}$ for $12-16 \mathrm{~h}$. After cooling 
down, the reaction was filtered and the polymer recovered and used directly in another run. The combined filtrate from 5 reaction runs was concentrated in vacuo. The resulting crude was diluted with ethyl acetate $(75 \mathrm{~mL})$ and washed with brine $(3 \times 20 \mathrm{~mL})$. The organic layer was dried with $\mathrm{MgSO}_{4}$ and concentrated in vacuo to afford $\mathbf{2 2}$ as a light yellow oil $(3.34 \mathrm{~g}, 100 \%) .{ }^{1} \mathrm{H} \mathrm{NMR}\left(\mathrm{CDCl}_{3}, 300 \mathrm{MHz}\right) \delta: 10.91(\mathrm{bs}, 1 \mathrm{H}, \mathrm{OH}), 6.94\left(\mathrm{~d},{ }^{1} J_{\mathrm{HP}}=550\right.$ $\mathrm{Hz}, 1 \mathrm{H}, \mathrm{H}-\mathrm{P}), 5.13(\mathrm{~m}, 1 \mathrm{H},-\mathrm{CH}=), 2.57\left(\mathrm{dd},{ }^{2} J_{\mathrm{HCP}}=19.0 \mathrm{~Hz},{ }^{3} J_{\mathrm{H}, \mathrm{H}}=8.0 \mathrm{~Hz}, 2 \mathrm{H},-\mathrm{CH}_{2^{-}}\right.$ P), $1.77\left(\mathrm{~d},{ }^{4} J_{\mathrm{H}, \mathrm{H}}=4.0 \mathrm{~Hz}, 3 \mathrm{H}, \mathrm{CH}_{3}-\mathrm{C}=\right), 1.66\left(\mathrm{~d},{ }^{4} J_{\mathrm{H}, \mathrm{H}}=4.0 \mathrm{~Hz}, 3 \mathrm{H}, \mathrm{CH}_{3}-\mathrm{C}=\right) ;{ }^{13} \mathrm{C} \mathrm{NMR}$ $\left(\mathrm{CDCl}_{3}, 75.45 \mathrm{MHz}\right) \delta: 138.4\left(\mathrm{~d}, J_{\mathrm{PCCC}}=14 \mathrm{~Hz}\right), 110.6\left(\mathrm{~d}, J_{\mathrm{PCC}}=9 \mathrm{~Hz}\right), 30.1\left(\mathrm{~d}, J_{\mathrm{PC}}=92\right.$ $\mathrm{Hz}), 25.8\left(\mathrm{~d}, J_{\mathrm{PCCCC}}=3 \mathrm{~Hz}\right), 18.2\left(\mathrm{~d}, J_{\mathrm{PCCCC}}=3 \mathrm{~Hz}\right) ;{ }^{31} \mathrm{P} \mathrm{NMR}\left(\mathrm{CDCl}_{3}, 121.47 \mathrm{MHz}\right) \delta$ : $35.86\left(\mathrm{dm}, J_{\mathrm{PH}}=550 \mathrm{~Hz}\right)$; HRMS $\left(\mathrm{EI}^{+}\right)$calcd. for $\mathrm{C}_{5} \mathrm{H}_{11} \mathrm{O}_{2} \mathrm{P},\left([\mathrm{M}]^{+}\right) 134.0497$, found 134.0494 .

\section{(1,1-Diethoxy-ethyl)-(3-methyl-but-2-enyl)-phosphinic acid ethyl ester 24 (Scheme 4, Table 2, Entry 3)}

Method A, 0.2 eq. $\mathrm{BF}_{3} \cdot \mathrm{OEt}_{2}, 24 \mathrm{~h}$. Chromatography over silica gel (hexanes / ethyl acetate, $7 / 3$, v/v) afforded 24 as a light yellow oil $\left(0.89 \mathrm{~g}, 64 \%\right.$ yield). ${ }^{1} \mathrm{H} \mathrm{NMR}\left(300 \mathrm{MHz}, \mathrm{CDCl}_{3}\right)$ ঠ: 5.22-5.29 (m, $1 \mathrm{H},-\mathrm{CH}=), 4.12-4.26\left(\mathrm{~m}, 2 \mathrm{H},-\mathrm{CH}_{2}-\mathrm{O}\right), 3.62-3.83\left(\mathrm{~m}, 4 \mathrm{H},-\mathrm{CH}_{2}-\mathrm{O}\right)$, $2.59\left(\mathrm{dd},{ }^{2} J_{\mathrm{HCP}}=15.5 \mathrm{~Hz},{ }^{3} J_{\mathrm{H}, \mathrm{H}}=8.0 \mathrm{~Hz}, 2 \mathrm{H},-\mathrm{CH}_{2}-\mathrm{P}\right), 1.77\left(\mathrm{~d},{ }^{4} J_{\mathrm{H}, \mathrm{H}}=4.0 \mathrm{~Hz}, 3 \mathrm{H}, \mathrm{CH}_{3}-\right.$ $\mathrm{C}=), 1.67\left(\mathrm{~d},{ }^{4} J_{\mathrm{H}, \mathrm{H}}=4.0 \mathrm{~Hz}, 3 \mathrm{H}, \mathrm{CH}_{3}-\mathrm{C}=\right), 1.51\left(\mathrm{~d},{ }^{3} J_{\mathrm{HCCP}}=11.0 \mathrm{~Hz}, 3 \mathrm{H}, \mathrm{CH}_{3}-\mathrm{C}-\mathrm{P}\right), 1.30$ $\left(\mathrm{t},{ }^{3} J_{\mathrm{H}, \mathrm{H}}=7.0 \mathrm{~Hz}, 3 \mathrm{H}, \mathrm{CH}_{3^{-}}\right), 1.22\left(\mathrm{t},{ }^{3} \mathrm{~J}_{\mathrm{H}, \mathrm{H}}=7.0 \mathrm{~Hz}, 6 \mathrm{H}, \mathrm{CH}_{3^{-}}\right) ;{ }^{13} \mathrm{C} \mathrm{NMR}(75.45 \mathrm{MHz}$, $\left.\mathrm{CDCl}_{3}\right) \delta: 136.5\left(\mathrm{~d}, J_{\mathrm{PCCC}}=12.0 \mathrm{~Hz}\right), 112.6\left(\mathrm{~d}, J_{\mathrm{PCC}}=9.5 \mathrm{~Hz}\right), 101.4\left(\mathrm{~d}, J_{\mathrm{PC}}=137.0 \mathrm{~Hz}\right)$, $61.7\left(\mathrm{~d}, J_{\mathrm{POC}}=7.0 \mathrm{~Hz}\right), 58.2\left(\mathrm{~d}, J_{\mathrm{PCOC}}=4.5 \mathrm{~Hz}\right), 57.7\left(\mathrm{~d}, J_{\mathrm{PCOC}}=7.0 \mathrm{~Hz}\right), 26.2\left(\mathrm{~d}, J_{\mathrm{PC}}=\right.$ $84.5 \mathrm{~Hz}), 25.9\left(\mathrm{~d}, J_{\mathrm{PCCCC}}=2.5 \mathrm{~Hz}\right), 20.6\left(\mathrm{~d}, J_{\mathrm{PCC}}=12.0 \mathrm{~Hz}\right), 18.1\left(\mathrm{~d}, J_{\mathrm{PCCCC}}=2.0 \mathrm{~Hz}\right)$, $16.7\left(\mathrm{~d}, J_{\mathrm{POCC}}=5.5 \mathrm{~Hz}\right), 15.3,15.6 ;{ }^{31} \mathrm{P} \mathrm{NMR}\left(121.47 \mathrm{MHz}, \mathrm{CDCl}_{3}\right) \delta: 47.30(\mathrm{~s}) ; \mathrm{HRMS}$ (Ammonium Chem Ion) calcd. for $\mathrm{C}_{13} \mathrm{H}_{28} \mathrm{O}_{4} \mathrm{P},\left([\mathrm{M}+\mathrm{H}]^{+}\right.$) 279.1725, found 279.1727.

\section{(1,1-Diethoxy-ethyl)-( $N$-acetyl-L-aspartic acid dibenzyl ester)-phosphinic acid ethyl ester 26 (Scheme 4)}

After saturation of a solution of the ester $\mathbf{2 4}\left(1.37 \mathrm{~g}, 4.93 \mathrm{mmol}, 1.0\right.$ equiv.) in $\mathrm{CH}_{2} \mathrm{Cl}_{2}(0.2$ $\mathrm{M}, 26.0 \mathrm{~mL})$ at $-78{ }^{\circ} \mathrm{C}$ with ozone, $\mathrm{Me}_{2} \mathrm{~S}(2.5 \mathrm{~mL}, 33.5 \mathrm{mmol}, 6.8$ equiv.) was added under $\mathrm{N}_{2}$ at $-78{ }^{\circ} \mathrm{C}$. The reaction mixture was allowed to warm up to r.t. overnight, then the solution was concentrated in vacuo. The resulting oil was dissolved in a mixture $t \mathrm{BuOH} /$ $\mathrm{H}_{2} \mathrm{O}(2 / 1, \mathrm{v} / \mathrm{v}, 20 \mathrm{~mL})$ and treated successively with 2-methyl-butene ( $0.5 \mathrm{M}$ in THF, 9.8 $\mathrm{mL}, 9.8 \mathrm{mmol}, 2.0$ equiv. $), \mathrm{NaH}_{2} \mathrm{PO}_{4} \bullet \mathrm{H}_{2} \mathrm{O}(1.0 \mathrm{~g}, 7.4 \mathrm{mmol}, 1.5$ equiv. $)$ and $\mathrm{NaClO}_{2}(0.67$ $\mathrm{g}, 3.4 \mathrm{mmol}, 1.5$ equiv.) at $0{ }^{\circ} \mathrm{C}$. After $3 \mathrm{~h}$ at r.t., the reaction mixture was diluted with ethyl acetate $(50 \mathrm{~mL})$ and washed with $10 \%$ aqueous tartaric acid $(2 \times 15 \mathrm{~mL})$. The aqueous layer was reextracted with ethyl acetate $(1 \times 20 \mathrm{~mL})$. The combined organic layers were dried over $\mathrm{MgSO}_{4}$ and concentrated in vacuo to afford compound $\mathbf{2 5}$ which was used without further purification.

To a solution of the compound 25 (4.93 mmol, 1.0 equiv.) in distilled THF ( $0.2 \mathrm{M}, 26 \mathrm{~mL}$ ) was added L-aspartic acid dibenzyl ester $p$-toluenesulfonate ( $2.38 \mathrm{~g}, 4.9 \mathrm{mmol}, 1.0$ equiv.), $\mathrm{Et}_{3} \mathrm{~N}(0.76 \mathrm{~mL}, 5.4 \mathrm{mmol}, 1.1$ equiv.), $\mathrm{EDC} \cdot \mathrm{HCl}(2.8 \mathrm{~g}, 14.7 \mathrm{mmol}, 3.0$ equiv.) and DMAP ( $2.38 \mathrm{~g}, 4.9 \mathrm{mmol}, 3.0$ equiv.). After $16 \mathrm{~h}$ at r.t. under $\mathrm{N}_{2}$, the reaction mixture was diluted with ethyl acetate $(50 \mathrm{~mL})$ and washed with $0.5 \mathrm{M}$ aqueous $\mathrm{HCl}(1 \times 10 \mathrm{~mL})$ and brine $(2 \times$ $20 \mathrm{~mL}$ ). The organic layer was dried over $\mathrm{MgSO}_{4}$ and concentrated in vacuo. The resulting oil was purified by chromatography over silica gel (hexanes / ethyl acetate, 6/4, v/v to ethyl acetate $100 \%$ ) to afford amide 26 as a lightly yellow oil ( $1.81 \mathrm{~g}, 66 \%$ yield). ${ }^{1} \mathrm{H}$ NMR (300 $\left.\mathrm{MHz}, \mathrm{CDCl}_{3}\right)$ d: $7.88 \& 7.75\left(2 \mathrm{~d},{ }^{3} J_{\mathrm{H}, \mathrm{H}}=8.0 \mathrm{~Hz}, 1 \mathrm{H}, \mathrm{NH}\right), 7.29-7.37(\mathrm{~m}, 10 \mathrm{H}$, aro $\mathrm{CH})$, $5.10 \& 5.09\left(2 \mathrm{~d},{ }^{2} J_{\mathrm{H}, \mathrm{H}}=12.0 \mathrm{~Hz}, 4 \mathrm{H}, B n-\mathrm{CH}_{2}-\right), 4.90-4.98(\mathrm{~m}, 1 \mathrm{H},-\mathrm{CH}-\mathrm{N}), 4.13-4.5$ (m, $\left.2 \mathrm{H},-\mathrm{CH}_{2}-\mathrm{O}\right), 3.59-3.81\left(\mathrm{~m}, 4 \mathrm{H},-\mathrm{CH}_{2}-\mathrm{O}\right), 2.74-3.08$ (m, $\left.4 \mathrm{H},-\mathrm{CH}_{2}-\&-\mathrm{CH}_{2}-\mathrm{P}\right), 1.49$ $\left(\mathrm{d},{ }^{3} \mathrm{~J}_{\mathrm{HCCP}}=12.0 \mathrm{~Hz}, 3 \mathrm{H}, \mathrm{CH}_{3}-\mathrm{C}-\mathrm{P}\right), 1.17-1.37(\mathrm{~m}, 9 \mathrm{H}) ;{ }^{13} \mathrm{C} \mathrm{NMR}\left(75.45 \mathrm{MHz}, \mathrm{CDCl}_{3}\right)$ 


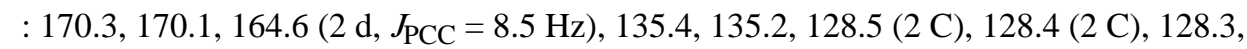
$101.0\left(\mathrm{~d}, J_{\mathrm{PC}}=150.5 \mathrm{~Hz}\right), 67.4,66.8,62.6\left(2 \mathrm{~d}, J_{\mathrm{POC}}=8.0 \mathrm{~Hz}\right), 58.5\left(2 \mathrm{~d}, J_{\mathrm{PCOC}}=5.0 \mathrm{~Hz}\right)$, $57.9\left(2 \mathrm{~d}, J_{\mathrm{PCOC}}=8.0 \mathrm{~Hz}\right), 49.1\left(\mathrm{~d}, J_{\mathrm{PCCNC}}=10.5 \mathrm{~Hz}\right), 36.4\left(\mathrm{~d}, J_{\mathrm{PCCNCC}}=6.0 \mathrm{~Hz}\right), 34.6$ \& $34.5\left(2 \mathrm{~d}, J_{\mathrm{PC}}=72.0 \mathrm{~Hz}\right), 20.0\left(\mathrm{~d}, J_{\mathrm{PCC}}=13.0 \mathrm{~Hz}\right), 16.5\left(2 \mathrm{~d}, J_{\mathrm{POCC}}=4.5 \mathrm{~Hz}\right), 15.3$, 15.6; ${ }^{31} \mathrm{P}$ NMR (121.47 MHz, $\mathrm{CDCl}_{3}$ ) $\delta: 42.77$ \& 42.70 (2 s); HRMS (Ammonium Chem Ion) calcd. for $\mathrm{C}_{28} \mathrm{H}_{38} \mathrm{NO}_{9} \mathrm{P},\left([\mathrm{M}+\mathrm{H}]^{+}\right)$564.2362, found 564.2355.

\section{(N-acetyl-L-aspartic acid)-H-phosphinic acid 27 (Scheme 4)}

To a solution of amide $26(1.0 \mathrm{~g}, 1.78 \mathrm{mmol})$ in a mixture $\mathrm{THF} / \mathrm{H}_{2} \mathrm{O}(2 / 1, \mathrm{v} / \mathrm{v}, 5.1 \mathrm{~mL})$ was added $\mathrm{Pd} / \mathrm{C}(0.24 \mathrm{~g}, 10 \mathrm{wt} \%)$. The reaction was placed in a hydrogenator at $50 \mathrm{psi} \mathrm{H}_{2}$ and r.t. After $17 \mathrm{~h}$, the reaction mixture was filtered through Celite ${ }^{\circledR}$ and concentrated in vacuo. The resulting oil was dissolved in water $(30 \mathrm{~mL})$ and washed with $\mathrm{CH}_{2} \mathrm{Cl}_{2}(3 \times 10 \mathrm{~mL})$. The aqueous layer was concentrated then dissolved in a mixture $\mathrm{THF} / \mathrm{H}_{2} \mathrm{O}(2 / 1, \mathrm{v} / \mathrm{v}, 12 \mathrm{~mL})$ and treated with washed Amberlite IR-120 plus $(0.83 \mathrm{~g})$. The suspension was heated at $80{ }^{\circ} \mathrm{C}$ for $15 \mathrm{~h}$ under $\mathrm{N}_{2}$. After filtration, the reaction mixture was concentrated in vacuo. The resulting oil was dissolved in water, neutralized at $\mathrm{pH} 7.0$ and purified by ion exchange over AG-1 $\mathrm{X} 8$ anion exchange resin $(70 \mathrm{~mL})$ which had been equilibrated with $100 \mathrm{mM} \mathrm{Et}_{3} \mathrm{NH}^{+} \mathrm{HCO}_{3}{ }^{-}$ $(\mathrm{pH}$ 7.3). The column was washed with water $(60 \mathrm{~mL})$ then eluted with a linear gradient $(200 \mathrm{~mL}+200 \mathrm{~mL}, 100-500 \mathrm{mM})$ of $\mathrm{Et}_{3} \mathrm{NH}^{+} \mathrm{HCO}_{3}{ }^{-}$(pH 7.3). Fractions containing phosphorus compounds were identified by using the Pi assay developed by $\mathrm{Ames}^{22}$ and concentrated. The resulting white residue was dissolved in water, the solution passed through a Dowex $\mathrm{H}^{+}$column, neutralized to $\mathrm{pH} 7$ and then concentrated to afford $\mathrm{H}$ phosphinic acid 27 as a light yellow oil $\left(0.243 \mathrm{~g}, 57 \%\right.$ yield). ${ }^{1} \mathrm{H}$ NMR $\left(300 \mathrm{MHz}, \mathrm{D}_{2} \mathrm{O}\right) \delta$ : $6.95\left(\mathrm{~d},{ }^{1} J_{\mathrm{HP}}=540.0 \mathrm{~Hz}, 1 \mathrm{H}, \mathrm{H}-\mathrm{P}\right), 4.22\left(\mathrm{dd},{ }^{3} J_{\mathrm{H}, \mathrm{H}}=9.0 \mathrm{~Hz},{ }^{3} J_{\mathrm{H}, \mathrm{H}}=4.0 \mathrm{~Hz}, 1 \mathrm{H},-\mathrm{CH}-\mathrm{N}\right)$, $2.47-2.58\left(\mathrm{~m}, 3 \mathrm{H},-\mathrm{CH}_{2}-\mathrm{P},-\mathrm{CH}_{2}-\right), 2.35\left(\mathrm{dd},{ }^{2} J_{\mathrm{H}, \mathrm{H}}=16.0 \mathrm{~Hz},{ }^{3} J_{\mathrm{H}, \mathrm{H}}=9.0 \mathrm{~Hz}, 1 \mathrm{H},-\right.$ $\left.\mathrm{CH}_{2}-\right) ;{ }^{13} \mathrm{C}$ NMR $\left(75.45 \mathrm{MHz}, \mathrm{D}_{2} \mathrm{O}\right) \delta: 179.0,178.8,168.9,53.6,41.5\left(\mathrm{~d}, J_{\mathrm{PC}}=78.5 \mathrm{~Hz}\right)$, $39.8 ;{ }^{31} \mathrm{P} \mathrm{NMR}\left(121.47 \mathrm{MHz}, \mathrm{D}_{2} \mathrm{O}\right) \delta: 20.76\left(\mathrm{dt}, J_{\mathrm{PH}}=539.5 \mathrm{~Hz}, J_{\mathrm{PCH}}=17.0 \mathrm{~Hz}\right) ; \mathrm{HRMS}$ $\left(\mathrm{ESI}^{+}\right)$calcd. for $\mathrm{C}_{6} \mathrm{H}_{10} \mathrm{O}_{7} \mathrm{NP},\left([\mathrm{M}+\mathrm{Na}]^{+}\right)$262.0093, found 262.0096 .

\section{(1,1-Diethoxy-ethyl)-(2-amino-2-cyano-ethyl)-phosphinic acid ethyl ester 28 (Scheme 5)}

After saturation of a solution of the ester $\mathbf{2 4}\left(0.834 \mathrm{~g}, 3.0 \mathrm{mmol}, 1.0\right.$ equiv.) in $\mathrm{CH}_{2} \mathrm{Cl}_{2}(0.2$ $\mathrm{M}, 15.0 \mathrm{~mL})$ at $-78{ }^{\circ} \mathrm{C}$ with ozone, $\mathrm{Me}_{2} \mathrm{~S}(1.5 \mathrm{~mL}, 20.4 \mathrm{mmol}, 6.8$ equiv. $)$ was added under $\mathrm{N}_{2}$ at $-78^{\circ} \mathrm{C}$. The reaction mixture was allowed to warm up to r.t. overnight then the solution was concentrated in vacuo. The resulting oil was dissolved in methanol $(0.8 \mathrm{M}, 4.0$ $\mathrm{mL}$ ) then added dropwise to a solution of sodium cyanide ( $0.25 \mathrm{~g}, 5.1 \mathrm{mmol}, 1.7$ equiv.), ammonium chloride $(0.284 \mathrm{~g}, 5.4 \mathrm{mmol}, 1.8$ equiv.) in $25 \%$ aqueous ammonium hydroxyde $(1.0 \mathrm{M}, 4.5 \mathrm{~mL})$ at $0^{\circ} \mathrm{C}$. The reaction mixture was kept at $0{ }^{\circ} \mathrm{C}$ for $1 \mathrm{~h}$ then at r.t. for $17 \mathrm{~h}$. The reaction mixture was extracted with ether $(3 \times 50 \mathrm{~mL})$ and the combined organic layers were washed with brine $(2 \times 20 \mathrm{~mL})$. The organic layer was dried with $\mathrm{MgSO}_{4}$ and concentrated in vacuo to afford amine 28 as a colorless oil $(0.566 \mathrm{~g}, 68 \%) .2$ diastereoisomers 54/46. ${ }^{1} \mathrm{H}$ NMR (300 MHz, $\left.\mathrm{CDCl}_{3}\right)$ \&: 4.19-4.35 (m, $2 \mathrm{H}+1 \mathrm{H},-\mathrm{CH}_{2}-\mathrm{O}$ \& -CH-N), 3.63-3.84 (m, $\left.4 \mathrm{H},-\mathrm{CH}_{2}-\mathrm{O}\right), 1.94-2.49\left(\mathrm{~m}, 2 \mathrm{H}+2 \mathrm{H},-\mathrm{CH}_{2}-\mathrm{P} \& \mathrm{NH}_{2}\right), 1.53$ (2 $\left.\mathrm{d},{ }^{3} J_{\mathrm{HCCP}}=11.5 \mathrm{~Hz}, 3 \mathrm{H}, \mathrm{CH}_{3}-\mathrm{C}-\mathrm{P}\right), 1.37\left(\mathrm{t},{ }^{3} J_{\mathrm{H}, \mathrm{H}}=7.0 \mathrm{~Hz}, 3 \mathrm{H}, \mathrm{CH}_{3^{-}}\right), 1.24 \& 1.23(2$ $\left.\mathrm{t},{ }^{3} \mathrm{~J}_{\mathrm{H}, \mathrm{H}}=7.0 \mathrm{~Hz}, 6 \mathrm{H}, \mathrm{CH}_{3^{-}}\right) ;{ }^{13} \mathrm{C} \mathrm{NMR}\left(75.45 \mathrm{MHz}, \mathrm{CDCl}_{3}\right) \delta: 121.4\left(\mathrm{~d}, J_{\mathrm{PCCC}}=14.0 \mathrm{~Hz}\right)$ $\& 121.3\left(\mathrm{~d}, J_{\mathrm{PCCC}}=12.0 \mathrm{~Hz}\right), 101.3\left(\mathrm{~d}, J_{\mathrm{PC}}=147.0 \mathrm{~Hz}\right) \& 101.2\left(\mathrm{~d}, J_{\mathrm{PC}}=146.0 \mathrm{~Hz}\right), 62.6$ $\left(\mathrm{d}, J_{\mathrm{POC}}=6.5 \mathrm{~Hz}\right) \& 62.4\left(\mathrm{~d}, J_{\mathrm{POC}}=7.0 \mathrm{~Hz}\right), 58.8\left(2 \mathrm{~d}, J_{\mathrm{PCOC}}=5.5 \mathrm{~Hz}\right), 58.2\left(\mathrm{~d}, J_{\mathrm{PCOC}}=\right.$ $7.0 \mathrm{~Hz}) \& 58.1\left(\mathrm{~d}, J_{\mathrm{PCOC}}=8.0 \mathrm{~Hz}\right), 38.9\left(\mathrm{~d}, J_{\mathrm{PCC}}=4.0 \mathrm{~Hz}\right) \& 38.5\left(\mathrm{~d}, J_{\mathrm{PCC}}=3.5 \mathrm{~Hz}\right), 32.1$ $\& 31.7\left(2 \mathrm{~d}, J_{\mathrm{PC}}=83.0 \mathrm{~Hz}\right), 20.1\left(\mathrm{~d}, J_{\mathrm{PCC}}=14.0 \mathrm{~Hz}\right) \& 19.9\left(\mathrm{~d}, J_{\mathrm{PCC}}=12.5 \mathrm{~Hz}\right), 16.8(\mathrm{~d}$, $\left.J_{\mathrm{POCC}}=5.5 \mathrm{~Hz}\right), 15.7,15.5 \& 15.4 ;{ }^{31} \mathrm{P}$ NMR $\left(121.47 \mathrm{MHz}, \mathrm{CDCl}_{3}\right) \delta: 44.87(\mathrm{~s}, 54 \%)$, $44.68(\mathrm{~s}, 46 \%)$. 
(2-Benzyloxycarbonylamino-2-cyano-ethyl)-(1,1-diethoxy-ethyl)-phosphinic acid ethyl ester 30 (Scheme 5)

To amine $28(1.2 \mathrm{~g}, 4.3 \mathrm{mmol})$ in THF $(0.2 \mathrm{M}, 22.0 \mathrm{~mL})$ was added pyridine $(0.42 \mathrm{~mL}, 5.18$ mmol, 1.2 equiv.) followed by benzylchloroformate $\left(0.61 \mathrm{~mL}, 4.13 \mathrm{mmol}, 1.0\right.$ equiv.) at $0^{\circ} \mathrm{C}$ under $\mathrm{N}_{2}$. After $3 \mathrm{~h}$ at r.t., the reaction mixture was diluted with ethyl acetate $(75 \mathrm{~mL})$ and washed with brine $(3 \times 20 \mathrm{~mL})$. The organic layer was dried with $\mathrm{MgSO}_{4}$ and concentrated in vacuo. The resulting oil was purified by chromatography over silica gel (hexanes / ethyl acetate, $7 / 3, \mathrm{v} / \mathrm{v})$ to afford protected amine $\mathbf{3 0}$ as a colorless oil $(1.58 \mathrm{~g}, 89 \%) .{ }^{1} \mathrm{H}$ NMR $\left(300 \mathrm{MHz}, \mathrm{CDCl}_{3}\right)$ \&: $7.35(\mathrm{~s}, 5 \mathrm{H}$, aro $\mathrm{CH}), 6.83\left(\mathrm{~d},{ }^{3} \mathrm{~J}_{\mathrm{H}, \mathrm{H}}=8.0 \mathrm{~Hz}, 0.54 \mathrm{H}, \mathrm{NH}\right), 6.49$ $\left(\mathrm{d},{ }^{3} \mathrm{~J}_{\mathrm{H}, \mathrm{H}}=6.5 \mathrm{~Hz}, 0.46 \mathrm{H}, \mathrm{NH}\right), 5.14\left(\mathrm{~s}, 2 \mathrm{H}, \mathrm{Cbz}-\mathrm{CH}_{2}-\right), 4.90-5.10(\mathrm{~m}, 1 \mathrm{H},-\mathrm{CH}-\mathrm{N})$, 4.08-4.35 (m, $\left.2 \mathrm{H},-\mathrm{CH}_{2}-\mathrm{O}\right), 3.55-3.82\left(\mathrm{~m}, 4 \mathrm{H},-\mathrm{CH}_{2}-\mathrm{O}\right), 2.23-2.52\left(\mathrm{~m}, 2 \mathrm{H},-\mathrm{CH}_{2}-\mathrm{P}\right), 1.51$ \& $1.48\left(2 \mathrm{~d},{ }^{3} \mathrm{~J}_{\mathrm{HCCP}}=11.5 \mathrm{~Hz}, 3 \mathrm{H}, \mathrm{CH}_{3} \mathrm{C}-\mathrm{P}\right), 1.34 \& 1.30\left(2 \mathrm{t},{ }^{3} \mathrm{~J}_{\mathrm{H}, \mathrm{H}}=7.0 \mathrm{~Hz}, 3 \mathrm{H}, \mathrm{CH}_{3^{-}}\right)$, 1.17-1.23 (m, $\left.6 \mathrm{H}, \mathrm{CH}_{3^{-}}\right) ;{ }^{13} \mathrm{C}$ NMR $\left(75.45 \mathrm{MHz}, \mathrm{CDCl}_{3}\right)$ ठ: 155.2, 135.7, $128.5(2 \mathrm{C})$, $128.3,128.2(2 \mathrm{C}), 118.0 \& 117.8\left(2 \mathrm{~d}, J_{\mathrm{PCCC}}=10.0 \mathrm{~Hz}\right), 100.9\left(\mathrm{~d}, J_{\mathrm{PC}}=148.5 \mathrm{~Hz}\right) \&$ $100.8\left(\mathrm{~d}, J_{\mathrm{PC}}=148.0 \mathrm{~Hz}\right), 67.5,62.7 \& 62.6\left(2 \mathrm{~d}, J_{\mathrm{POC}}=7.0 \mathrm{~Hz}\right), 58.8 \& 58.7\left(2 \mathrm{~d}, J_{\mathrm{PCOC}}=\right.$ $5.5 \mathrm{~Hz}), 58.2 \& 58.1\left(2 \mathrm{~d}, J_{\mathrm{PCOC}}=7.5 \mathrm{~Hz}\right), 38.0\left(\mathrm{~d}, J_{\mathrm{PCC}}=4.0 \mathrm{~Hz}\right), 28.9 \& 27.9\left(2 \mathrm{~d}, J_{\mathrm{PC}}=\right.$ $82.0 \mathrm{~Hz}), 19.6 \& 19.5\left(2 \mathrm{~d}, J_{\mathrm{PCC}}=12.0 \mathrm{~Hz}\right), 16.5\left(2 \mathrm{~d}, J_{\mathrm{POCC}}=5.5 \mathrm{~Hz}\right), 15.4,15.2 \&$ $15.1 ;{ }^{31} \mathrm{P}$ NMR (121.47 MHz, $\left.\mathrm{CDCl}_{3}\right) \delta: 45.75$ (s, $\left.54 \%\right), 43.82$ (s, $\left.46 \%\right)$; HRMS (Ammonium Chem Ion) calcd. for $\mathrm{C}_{19} \mathrm{H}_{30} \mathrm{~N}_{2} \mathrm{O}_{6} \mathrm{P},\left([\mathrm{M}+\mathrm{H}]^{+}\right)$413.1842, found 413.1839.

\section{(2-Benzyloxycarbonylamino-2-cyano-ethyl)-phenyl-phosphinic acid ethyl ester 31 (Scheme} 5)

To the acetal $30(0.412 \mathrm{~g}, 1.0 \mathrm{mmol})$ in chloroform $(0.1 \mathrm{M}, 10.0 \mathrm{~mL})$ was added chlorotrimethylsilane $(0.25 \mathrm{~mL}, 2.0 \mathrm{mmol}, 2.0$ equiv. $)$ at r.t. under $\mathrm{N}_{2}$. After $4 \mathrm{~h}$, the reaction mixture was diluted with chloroform $(50 \mathrm{~mL})$ and washed with $0.5 \mathrm{M}$ aqueous $\mathrm{NaHCO}_{3}(1 \times 20 \mathrm{~mL})$ and brine $(2 \times 20 \mathrm{~mL})$. The organic layer was dried with $\mathrm{MgSO}_{4}$ and concentrated in vacuo to afford the $H$-phosphinic ethyl ester as a colorless oil, which was used without further purification. To the $H$-phosphinic ethyl ester in acetonitrile $(0.1 \mathrm{M}, 10.0$ $\mathrm{mL}$ ) was added diisopropylethylamine $(0.23 \mathrm{~mL}, 1.3 \mathrm{mmol}, 1.3$ equiv.), iodobenzene $(0.11$ $\mathrm{mL}, 1.0 \mathrm{mmol}, 1.0$ equiv.) followed by $\mathrm{Pd}(\mathrm{OAc})_{2}(0.0045 \mathrm{mg}, 0.02 \mathrm{mmol}, 2.0 \mathrm{~mol}-\%)$ and dppf (0.0122 mg, $0.022 \mathrm{mmol}, 2.2 \mathrm{~mol}-\%)$. After $2 \mathrm{~h}$ at reflux under $\mathrm{N}_{2}$, the reaction mixture was cooled down and another portion of $\mathrm{Pd}(\mathrm{OAc})_{2}(0.0045 \mathrm{mg}, 0.02 \mathrm{mmol}, 2.0$ mol-\%) and dppf $(0.0122 \mathrm{mg}, 0.022 \mathrm{mmol}, 2.2 \mathrm{~mol}-\%)$ was added. After a total of $16 \mathrm{~h}$ at reflux under $\mathrm{N}_{2}$, the reaction mixture was cooled down and concentrated in vacuo. The resulting oil was dissolved with ethyl acetate $(50 \mathrm{~mL})$ and washed with brine $(3 \times 20 \mathrm{~mL})$. The organic layer was dried with $\mathrm{MgSO}_{4}$ and concentrated in vacuo. The resulting oil was purified by chromatography over silica gel (hexanes / ethyl acetate, $1 / 1, \mathrm{v} / \mathrm{v}$ ) to afford the phosphinic acid ethyl ester 31 as a colorless oil $(0.16 \mathrm{~g}, 43 \%) .{ }^{1} \mathrm{H}$ NMR $\left(300 \mathrm{MHz}, \mathrm{CDCl}_{3}\right)$ ઈ: 7.72-7.82 (m, $2 \mathrm{H}$, aro $\mathrm{CH}), 7.57-7.64(\mathrm{~m}, 1 \mathrm{H}$, aro $\mathrm{CH}), 7.50-7.52(\mathrm{~m}, 2 \mathrm{H}$, aro $\mathrm{CH})$; $7.32-7.36(\mathrm{~m}, 5 \mathrm{H}$, aro $\mathrm{CH}), 6.84\left(\mathrm{~d},{ }^{3} J_{\mathrm{H}, \mathrm{H}}=6.5 \mathrm{~Hz}, 0.5 \mathrm{H}, \mathrm{NH}\right), 6.71\left(\mathrm{~d},{ }^{3} J_{\mathrm{H}, \mathrm{H}}=6.5 \mathrm{~Hz}\right.$, $0.5 \mathrm{H}, \mathrm{NH}), 4.85-5.13$ (m, $\left.3 \mathrm{H}, \mathrm{Cbz}-\mathrm{CH}_{2}-\&-\mathrm{CH}-\mathrm{N}\right), 3.83-4.19$ (m, $\left.2 \mathrm{H},-\mathrm{CH}_{2}-\mathrm{O}\right), 2.22-$ $2.66\left(\mathrm{~m}, 2 \mathrm{H},-\mathrm{CH}_{2}-\mathrm{P}\right), 1.32 \& 1.29\left(2 \mathrm{~d},{ }^{3} J_{\mathrm{H}, \mathrm{H}}=7.0 \mathrm{~Hz}, 3 \mathrm{H}, \mathrm{CH}_{3^{-}}\right) ;{ }^{13} \mathrm{C} \mathrm{NMR}(75.45$ $\left.\mathrm{MHz}, \mathrm{CDCl}_{3}\right) \delta: 155.4 \& 155.3,136.0 \& 135.9,133.5\left(2 \mathrm{~d}, J_{\mathrm{PCCCC}}=6.5 \mathrm{~Hz}\right), 132.0 \&$ $131.7\left(2 \mathrm{~d}, J_{\mathrm{PCC}}=10.5 \mathrm{~Hz}, 2 \mathrm{C}\right), 129.5\left(\mathrm{~d}, J_{\mathrm{PC}}=128.5 \mathrm{~Hz}\right), 129.3 \& 129.2\left(2 \mathrm{~d}, J_{\mathrm{PCCC}}=\right.$ $13.0 \mathrm{~Hz}, 2 \mathrm{C}), 128.8(2 \mathrm{C}), 128.6,128.5(2 \mathrm{C}), 117.8\left(\mathrm{~d}, J_{\mathrm{PCCC}}=12.0 \mathrm{~Hz}\right) \& 117.6(\mathrm{~d}$, $\left.J_{\mathrm{PCCC}}=14.0 \mathrm{~Hz}\right), 67.7\left(\mathrm{~d}, J_{\mathrm{POC}}=7.0 \mathrm{~Hz}\right), 62.0 \& 61.8\left(\mathrm{~d}, J_{\mathrm{PCC}}=5.5 \mathrm{~Hz}\right), 38.3 \& 38.2,32.6$ $\left(\mathrm{d}, J_{\mathrm{PC}}=99.0 \mathrm{~Hz}\right) \& 32.4\left(\mathrm{~d}, J_{\mathrm{PC}}=100.0 \mathrm{~Hz}\right), 16.6\left(\mathrm{~d}, J_{\mathrm{POCC}}=6.0 \mathrm{~Hz}\right) ;{ }^{31} \mathrm{P} \mathrm{NMR}(121.47$ $\mathrm{MHz}, \mathrm{CDCl}_{3}$ ) $\delta: 40.55$ (s, $50 \%$ ), 39.79 (s, $50 \%$ ); HRMS (EI+) calcd. for $\mathrm{C}_{19} \mathrm{H}_{21} \mathrm{~N}_{2} \mathrm{O}_{4} \mathrm{P}$, $\left([\mathrm{M}]^{+}\right) 372.1239$, found 372.1241 . 
2-Amino-3-(hydroxy-phenyl-phosphinoyl)-propionic acid hydrochloride $32^{[17]}$ (Scheme 5)

A solution of phosphinate $\mathbf{3 1}(0.072 \mathrm{~g}, 0.19 \mathrm{mmol})$ in concentrated $\mathrm{HCl}(5.0 \mathrm{~mL})$ was refluxed under nitrogen. After $20 \mathrm{~h}$, the reaction mixture was cooled down and concentrated. The resulting oil was dissolved with water $(30 \mathrm{~mL})$ and washed with dichloromethane $(3 \times$ $10 \mathrm{~mL})$. The aqueous layer was concentrated to afford phosphinic acid $32(0.046 \mathrm{mg}, 90$ \%). ${ }^{1} \mathrm{H}$ NMR (300 MHz, $\left.\mathrm{D}_{2} \mathrm{O}\right) \delta: 7.56-7.63(\mathrm{~m}, 2 \mathrm{H}$, aro $\mathrm{CH}), 7.34-7.48(\mathrm{~m}, 3 \mathrm{H}$, aro $\mathrm{CH})$, $3.98\left(\mathrm{ddd},{ }^{3} J_{\mathrm{HCCP}}=15.0 \mathrm{~Hz}, 1 \mathrm{H},-\mathrm{CH}-\mathrm{N}\right), 2.36\left(\mathrm{ddd},{ }^{2} J_{\mathrm{HCP}}=14.0 \mathrm{~Hz},{ }^{2} J_{\mathrm{H}, \mathrm{H}}=13.5\right.$ $\left.\mathrm{Hz},{ }^{3} J_{\mathrm{H}, \mathrm{H}}=5.0 \mathrm{~Hz}, 1 \mathrm{H},-\mathrm{CH}_{2}-\mathrm{P}\right), 2.13\left(\mathrm{ddd},{ }^{2} J_{\mathrm{HCP}}=14.0 \mathrm{~Hz},{ }^{2} J_{\mathrm{H}, \mathrm{H}}=13.5 \mathrm{~Hz},{ }^{3} J_{\mathrm{H}, \mathrm{H}}=8.5\right.$ $\left.\mathrm{Hz}, 1 \mathrm{H},-\mathrm{CH}_{2}-\mathrm{P}\right) ;{ }^{13} \mathrm{C}$ NMR $\left(75.45 \mathrm{MHz}, \mathrm{D}_{2} \mathrm{O}\right) \delta: 171.1\left(\mathrm{~d}, J_{\mathrm{PCCC}}=11.0 \mathrm{~Hz}\right), 133.4\left(\mathrm{~d}, J_{\mathrm{PC}}\right.$ $=131.0 \mathrm{~Hz}), 132.3\left(\mathrm{~d}, J_{\mathrm{PCCCC}}=2.5 \mathrm{~Hz}\right), 131.0\left(\mathrm{~d}, J_{\mathrm{PCC}}=10.0 \mathrm{~Hz}, 2 \mathrm{C}\right), 128.9\left(\mathrm{~d}, J_{\mathrm{PCCC}}=\right.$ $12.5 \mathrm{~Hz}, 2 \mathrm{C}), 49.0,30.7\left(\mathrm{~d}, J_{\mathrm{PC}}=92.0 \mathrm{~Hz}\right) ;{ }^{31} \mathrm{P} \mathrm{NMR}\left(121.47 \mathrm{MHz}, \mathrm{CDCl}_{3}\right) \delta: 31.09(\mathrm{~s})$.

\section{4-(But-2-enoic acid tert-butyl ester)-(1,1-diethoxy-ethyl)-phosphinic acid ethyl ester 33 (Equation 3)}

To alkene $10(0.25 \mathrm{~g}, 1.0 \mathrm{mmol})$ in dichloromethane $(0.02 \mathrm{M}, 20.0 \mathrm{~mL})$ was added tert-butyl acrylate $(0.44 \mathrm{~mL}, 3.0 \mathrm{mmol}, 3.0$ equiv. $)$ and $2^{\text {nd }}$ generation Grubbs catalyst $34(0.042 \mathrm{~g}$, $0.05 \mathrm{mmol}, 5 \mathrm{~mol}-\%)$ at r.t.. After $22 \mathrm{~h}$, at reflux under $\mathrm{N}_{2}$, the reaction mixture was cooled down. Charcoal ( $2 \mathrm{~g})$ was added and the suspension was filtered through Celite ${ }^{\circledR}$. The solution was concentrated in vacuo. The resulting oil was purified by chromatography over silica gel (hexanes / ethyl acetate, 6/4, v/v) to afford ester 33 as a colorless oil $(0.257 \mathrm{~g}, 73$ \%). ${ }^{1} \mathrm{H}$ NMR (300 MHz, $\left.\mathrm{CDCl}_{3}\right)$ \&: 6.80-6.92 (m, $\left.1 \mathrm{H},-\mathrm{CH}=\right), 5.85-5.92(\mathrm{~m}, 1 \mathrm{H},-\mathrm{CH}=)$, 4.10-4.32 (m, $2 \mathrm{H},-\mathrm{CH}_{2}-\mathrm{O}$ ), 3.60-3.81 (m, $\left.4 \mathrm{H},-\mathrm{CH}_{2}-\mathrm{O}\right), 2.67-2.87$ (m, $\left.2 \mathrm{H},-\mathrm{CH}_{2}-\mathrm{P}\right), 1.53$ $\left(\mathrm{d},{ }^{3} J_{\mathrm{HCCP}}=12.0 \mathrm{~Hz}, 3 \mathrm{H}, \mathrm{CH}_{3}-\mathrm{C}-\mathrm{P}\right), 1.49\left(\mathrm{~s}, 9 \mathrm{H}, \mathrm{C}\left(\mathrm{CH}_{3}\right)_{3}\right), 1.34\left(\mathrm{t},{ }^{3} J_{\mathrm{H}, \mathrm{H}}=7.0 \mathrm{~Hz}, 3 \mathrm{H}\right.$, $\left.\mathrm{CH}_{3^{-}}\right), 1.23\left(\mathrm{t},{ }^{3} J_{\mathrm{H}, \mathrm{H}}=7.0 \mathrm{~Hz}, 3 \mathrm{H}, \mathrm{CH}_{3^{-}}\right), 1.22\left(\mathrm{t},{ }^{3} J_{\mathrm{H}, \mathrm{H}}=7.0 \mathrm{~Hz}, 3 \mathrm{H}, \mathrm{CH}_{3^{-}}\right) ;{ }^{13} \mathrm{C} \mathrm{NMR}$ $\left(75.45 \mathrm{MHz}, \mathrm{CDCl}_{3}\right) \delta: 165.2,136.6\left(\mathrm{~d}, J_{\mathrm{PCC}}=9.0 \mathrm{~Hz}\right), 127.6\left(\mathrm{~d}, J_{\mathrm{PCCC}}=11.0 \mathrm{~Hz}\right), 101.5$ $\left(\mathrm{d}, J_{\mathrm{PC}}=143.0 \mathrm{~Hz}\right), 80.6,62.2\left(\mathrm{~d}, J_{\mathrm{POC}}=7.0 \mathrm{~Hz}\right), 58.5\left(\mathrm{~d}, J_{\mathrm{PCOC}}=4.5 \mathrm{~Hz}\right), 58.0\left(\mathrm{~d}, J_{\mathrm{PCOC}}\right.$ $=7.0 \mathrm{~Hz}), 31.1\left(\mathrm{~d}, J_{\mathrm{PC}}=80.0 \mathrm{~Hz}\right), 28.3,20.5\left(\mathrm{~d}, J_{\mathrm{PCC}}=12.5 \mathrm{~Hz}\right), 16.8\left(\mathrm{~d}, J_{\mathrm{POCC}}=5.0 \mathrm{~Hz}\right)$, 15.7, 15.4; ${ }^{31} \mathrm{P}$ NMR (121.47 MHz, $\mathrm{CDCl}_{3}$ ) $\delta: 44.57$ (s); HRMS (Ammonium Chem Ion) calcd. for $\mathrm{C}_{16} \mathrm{H}_{32} \mathrm{O}_{6} \mathrm{P},\left([\mathrm{M}+\mathrm{H}]^{+}\right) 351.1937$, found 351.1941.

\section{Supplementary Material}

Refer to Web version on PubMed Central for supplementary material.

\section{Acknowledgments}

We thank the National Institute of General Medical Sciences/NIH (1R01 GM067610) for the financial support of this research.

\section{References}

1. (a) Gallagher MJ, Honegger H. Tetrahedron Lett. 1977; 18:2987-2990.(b) Gallagher MJ, Honegger H. Aust J Chem. 1980; 33:287-294.(c) Dingwall JG, Ehrenfreund J, Hall RG, Jack J. Phosphorus Sulfur. 1987; 30:571-574.(d) Dingwall JG, Ehrenfreund J, Hall RG. Tetrahedron. 1989; 45:37873808.(e) Baylis EK. Tetrahedron Lett. 1995; 36:9385-9388.(f) Baylis EK. Tetrahedron Lett. 1995; 36:9389-9392.(g) Froestl W, Mickel SJ, Hall RG, von Sprecher G, Strub D, Baumann DPA, Brugger F, Gentsch C, Jaekel J, Olpe HR, Rihs G, Vassout A, Waldmeier PC, Bittiger H. J Med Chem. 1995; 38:3297-3312. [PubMed: 7650684]

2. For some representative examples, see: McCleery PP, Tuck B. J Chem Soc Perkin Trans I. 1989:1319-1329.Froestl W, Mickel SJ, von Sprecher G, Diel PJ, Hall RG, Maier L, Strub D, Melillo V, Baumann PA, Bernasconi R, Gentsch C, Hauser K, Jaekel J, Karlsson G, Klebs K, Maitre L, Marescaux C, Pozza MF, Schmutz M, Steinmann MW, van Riezen H, Vassout A, Mondadori C, Olpe HR, Waldmeier PC, Bittiger H. J Med Chem. 1995; 38:3313-3331. [PubMed: 
7650685] Bennett SNL, Hall RG. J Chem Soc Trans 1. 1995:1145-1151.Belley M, Sullivan R, Reeves A, Evans J, O’Neill G, Ng GYK. Bioorg Med Chem. 1999; 7:2697-2704. [PubMed: 10658574] Walker CV, Caravatti G, Denholm AA, Egerton J, Faessler A, Furet P, GarciaEcheverria C, Gay B, Irving E, Jones K, Lambert A, Press NJ, Woods J. Bioorg Med Chem Lett. 2000; 10:2343-2346. [PubMed: 11055352] Reck F, Marmor S, Fisherb S, Wuonolaa MA. Bioorg Med Chem Lett. 2001; 11:1451-1454. [PubMed: 11378375] Hall RG, Riebli P. Phosphorus, Sulfur and Silicon. 2002; 177:1557-1562. Yamagishi T, Miyamae T, Yokomatsu T, Shibuya S. Tetrahedron Lett. 2004; 45:6713-6716.Prishchenko AA, Livantsov MV, Novikova OP, Livantsova LI. Russ J Gen Chem. 2006; 76:1963-1965.Yamagishi T, Haruki T, Yokomatsu T. Tetrahedron. 2006; 62:9210-9217. Yamagishi T, Haruki T, Yokomatsu T. Tetrahedron Asym. 2007; 18:28862893.Abrunhosa-Thomas I, Sellers CE, Montchamp JL. J Org Chem. 2007; 72:2851-2856. [PubMed: 17352490] Alstermark C, Amin K, Dinn SR, Elebring T, Fjellstrom O, Fitzpatrick K, Geiss WB, Gottfries J, Guzzo PR, Harding JP, Holmén A, Kothare Mohit, Lehmann A, Mattsson JP, Nilsson K, Sundén G, Swanson M, von Unge S, Woo AM, Wyle MJ, Zheng X. J Med Chem. 2008; 51:4315-4320. [PubMed: 18578471] Zhang D, Yuan C. Chem Eur J. 2008; 14:6049-6052. [PubMed: 18491337]

3. Reviews: Montchamp JL. Specialty Chemicals Magazine. 2006; 26:44-46.Montchamp JL. J Organomet Chem. 2005; 690:2388-2406.Coudray L, Montchamp J-L. Eur J Org Chem. 2008:36013613.Cross-Coupling: Montchamp JL, Dumond YR. J Am Chem Soc. 2001; 123:510-511.Dumond YR, Montchamp JL. J Organomet Chem. 2002; 653:252-260.Bravo-Altamirano K, Huang Z, Montchamp JL. Tetrahedron. 2005; 61:6315-6329.Bravo-Altamirano K, Montchamp JL. Org Lett. 2006; 8:4169-4171. [PubMed: 16928101] Bravo-Altamirano K, Montchamp JL. Tetrahedron Lett. 2007; 48:5755-5759. [PubMed: 17940584] Coudray L, Montchamp JL. Eur J Org Chem. 2008:4101-4103.Hydrophosphinylation: Deprèle S, Montchamp JL. J Org Chem. 2001; 66:67456755. [PubMed: 11578230] Deprèle S, Montchamp JL. J Am Chem Soc. 2002; 124:9386-9387. [PubMed: 12167029] Deprèle S, Montchamp JL. Org Lett. 2004; 6:3805-3808. [PubMed: 15469354] Ribière P, Bravo-Altamirano K, Antczak MI, Hawkins JD, Montchamp JL. J Org Chem. 2005; 70:4064-4072. [PubMed: 15876098] Bravo-Altamirano K, Abrunhosa-Thomas I, Montchamp JL. J Org Chem. 2008; 73:2292-2301. [PubMed: 18275217]

4. Belabassi Y, Antczak MI, Tellez J, Montchamp JL. Tetrahedron. 2008; 64:9181-9190. [PubMed: 19777052]

5. Stano A, Mucha A, Kafarski P. Synth Commun. 1999; 29:4269-4277.

6. J.-L. Montchamp, unpublished results. The reaction of $\operatorname{EtOP}(\mathrm{O}) \mathrm{H}_{2}$ with ketene diethyl acetal in refluxing acetonitrile gives 2 in $70 \%$ yield. Of course, this method is not competitive with the original Ciba-Geigy synthesis of 2.

7. Moskva VV, Maikova AI, Razumov AI. Russian J Gen Chem. 1969; 39:595-599.Livantsov MV, Prishchenko AA, Lutsenko IF. Zh Obshch Khim. 1987; 57:1042-1051.Endova M, Masojidkova M, Bucesinsky M, Rosenberg I. Tetrahedron Lett. 1996; 37:3497-3500.Ebetino FH, Berk JD. J Organomet Chem. 1997; 529:135-142.Luke GP, Shakespeare WC. Synth Commun. 2002; 32:2951-2957.Prishchenko AA, Livantsov MV, Novikova ON, Livantsova LI, Koval' Ya N, Grigor'ev EV. Russ J Gen Chem. 2004; 74:1317-1320.(g) See also ref. 1c.

8. Livantsov MV, Proskurnina MV, Prishchenko AA, Lutsenko IF. J Gen Chem USSR. 1984; 54:2237-2249.

9. Moskva VV, Maikova AI, Razumov AI. J Gen Chem USSR. 1969; 39:2391-2393.

10. Goldwhite H, Rowsell DG. J Am Chem Soc. 1966; 88:3572-3577. [PubMed: 5946163]

11. Coudray L, Kantrowitz ER, Montchamp JL. Bioorg Med Chem Lett. 2009; 19:900-902. [PubMed: 19097895]

12. The reactions between $\mathrm{H}$-phosphinic acids and triethyl orthoacetate or triethyl orthoformate were investigated in our original esterification work. Since some P-C bond formation was observed, yet the yields of either $\mathrm{H}$-phosphinate ester or acetal protected phosphinate were not very good and mixtures were obtained. This ultimately led to our discovery of the alkoxysilane-promoted esterification: Dumond YR, Baker RL, Montchamp JL. Org Lett. 2000; 2:3341-3344. [PubMed: 11029205]

13. Kolb HC, VanNieuwenhze MS, Sharpless KB. Chem Rev. 1994; 94:2483-2547. 
14. (a) Nelson DW, Gypser A, Ho PT, Kolb HC, Kondo T, Kwong HL, McGrath DV, Rubin AE, Norrby PO, Gable KP, Sharpless KB. J Am Chem Soc. 1997; 119:1840-1858.(b) Corey EJ, Guzman-Perez A, Noe MC. J Am Chem Soc. 1995; 117:10805-10816.

15. Raach A, Reiser O. J Prakt Chem. 2000; 342:605-608.

16. Khomutov AR, Osipova TI, Khurs EN, Alferov KV, Khomutov RM. Russ Chem Bull. 1996; 45:1961-1964.(b) See also ref. 1d.

17. Ross FC, Botting NP, Leeson PD. Bioorg Med Chem Lett. 1996; 6:2643-2646.

18. Formation of aryl phosphinate 31 proceeds in low yield and was sluggish. The cross-coupling of $H$-phosphinate esters is known in the literature and typically uses 5 mol-\% Pd or more. We elected to use dppf as the ligand since, in our hands, it gives better results than $\mathrm{PPh}_{3}$.

19. For example: Reviews: McReynolds MD, Dougherty JM, Hanson PR. Chem Rev. 2004; 104:2239-2258. [PubMed: 15137790] Diver ST, Giessert AJ. Chem Rev. 2004; 104:1317-1382. [PubMed: 15008625] General: Nawara AJ, Shima T, Hampel F, Gladysz JA. J Am Chem Soc. 2006; 128:4962-4963. [PubMed: 16608324] Dunne KS, Bisaro F, Odell B, Paris JM, Gouverneur V. J Org Chem. 2005; 70:10803-10809. [PubMed: 16356003] Ogasawara M, Nagano T, Hayashi T. Organometallics. 2003; 22:1174-1176.Ahmed M, Atkinson CE, Barrett AGM, Malagu K, Procopiou PA. Org Lett. 2003; 5:669-672. [PubMed: 12605486] Sprott KT, McReynolds MD, Hanson PR. Org Lett. 2001; 3:3939-3942. [PubMed: 11720574] Stoianova DS, Hanson PR. Org Lett. 2001; 3:3285-3288. [PubMed: 11594815] Stoianova DS, Hanson PR. Org Lett. 2000; 2:1769-1772. [PubMed: 10880222] Briot A, Bujard M, Gouverneur V, Nolan SP, Mioskowski C. Org Lett. 2000; 2:1517-1519. [PubMed: 10841468] Allcock HR, Laredo WR, deDenus CR, Taylor JP. Macromolecules. 1999; 32:7719-7725.Bujard M, Gouverneur V, Mioskowski C. J Org Chem. 1999; 64:2119-2123. [PubMed: 11674310] Sieck SR, McReynolds MD, Schroeder CE, Hanson PR. J Organomet Chem. 2006; 691:5307-5311.Coudray L, Bravo-Altamirano K, Montchamp JL. Org Lett. 2008; 10:1123-1126. [PubMed: 18288851] (r) see also refs 3n.

20. Cross-metathesis of phosphorus-containing compounds: Lera M, Hayes CJ. Org Lett. 2001; 3:2765-2768. [PubMed: 11506629] Demchuk OM, Pietrusiewicz KM, Michrowska A, Grela K. Org Lett. 2003; 5:3217-3220. [PubMed: 12943391] Chatterjee AK, Sanders DP, Grubbs RH. Org Lett. 2002; 4:1939. [PubMed: 12027652] He A, Yan B, Thanavaro A, Spilling CD, Rath NP. J Org Chem. 2004; 69:8643-8651. [PubMed: 15575740]

21. Bravo-Altamirano K, Montchamp JL. Org Synth. 2008; 85:96-105.

22. Ames BN. Meth Enzymol. 1966; 8:115-118. 

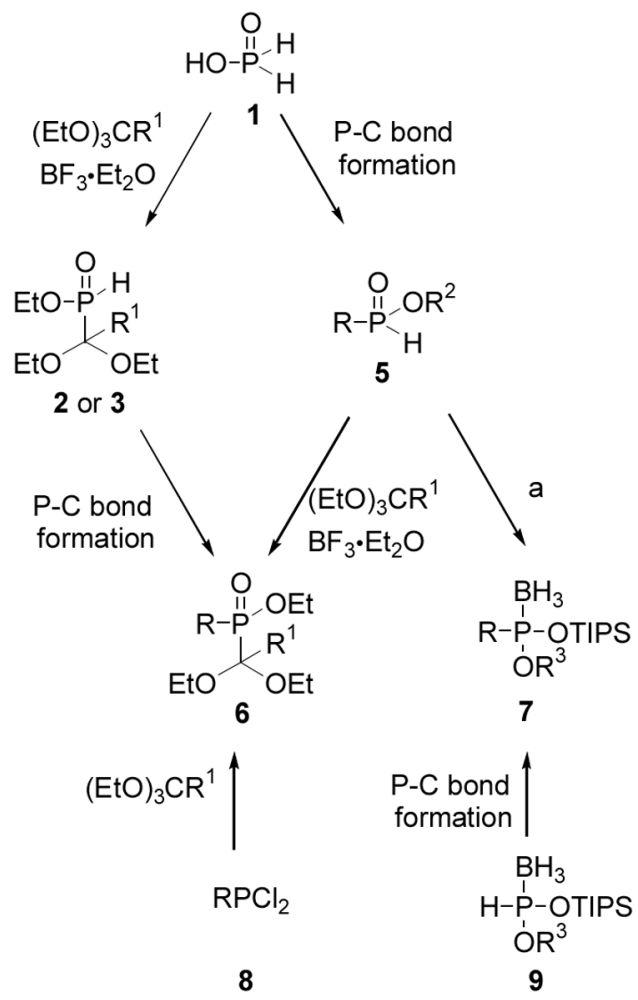

Scheme 1.

Strategies for the synthesis of protected phosphinates. $\mathrm{R}^{1}=\mathrm{H}, \mathrm{Me} ; \mathrm{R}^{2}=\mathrm{H}, \mathrm{Et} ; \mathrm{R}^{3}=\mathrm{TIPS}$, Et. [a] (i) TIPSCl/ $\mathrm{Et}_{3} \mathrm{~N}$ (1.5/1.6 equiv.), $\mathrm{CH}_{3} \mathrm{CN}, 0{ }^{\circ} \mathrm{C}$ to r.t., $14 \mathrm{~h}$; (ii) $\mathrm{BH}_{3} \bullet \mathrm{Me}_{2} \mathrm{~S}(2.0$ equiv.), $\mathrm{CH}_{3} \mathrm{CN}$, r.t., $5 \mathrm{~h}$. 

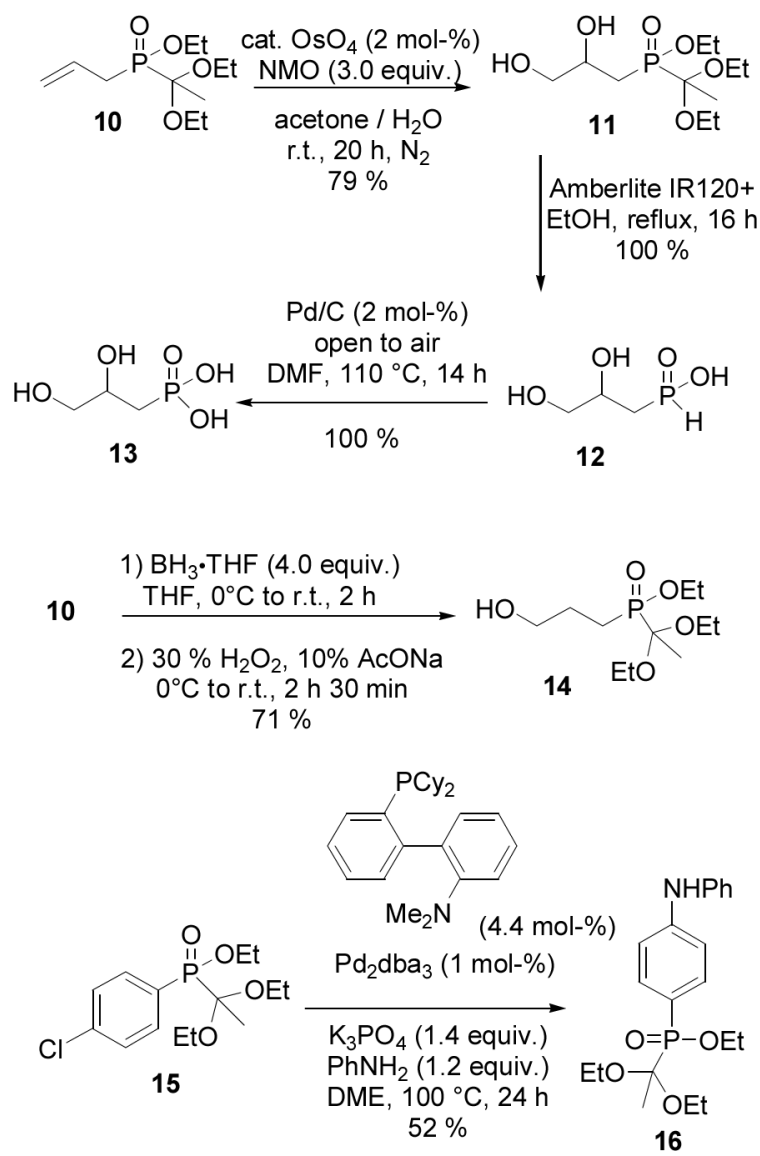

Scheme 2.

Dihydroxylation, hydroboration, and Buchwald-Hartwig amination. 

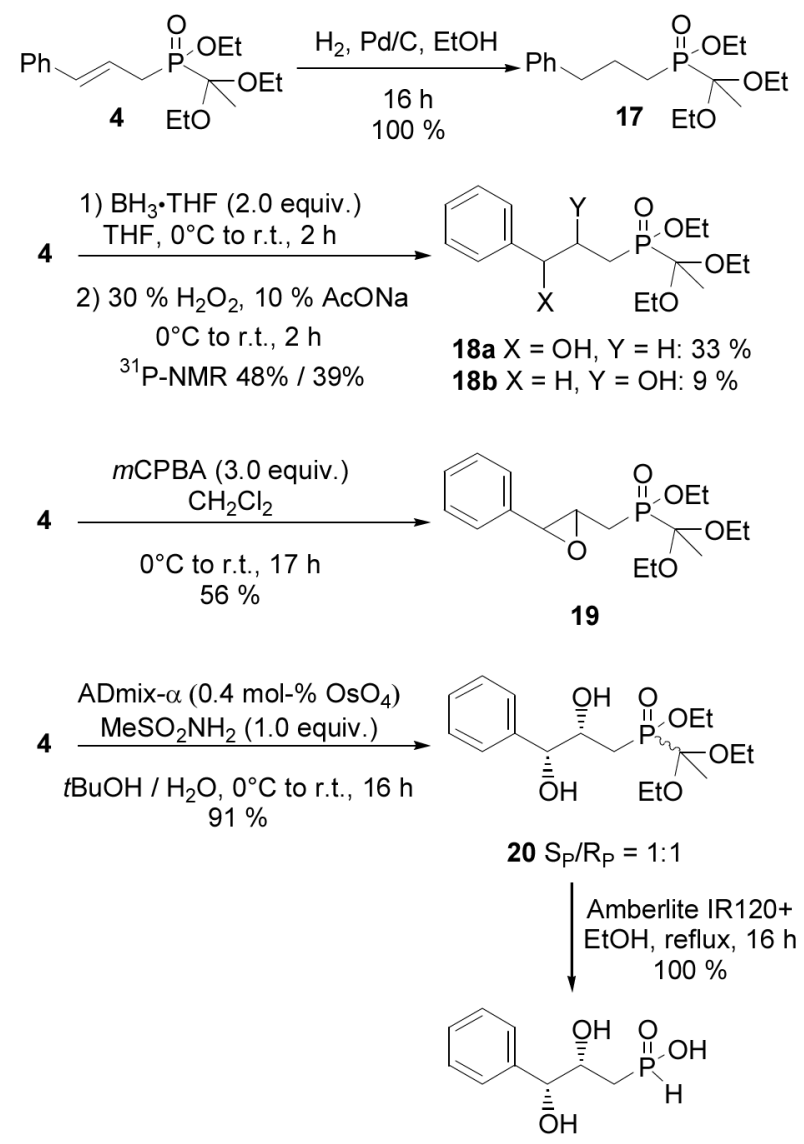

$2159 \%$ ee

Scheme 3.

Reactions on compound 4. 

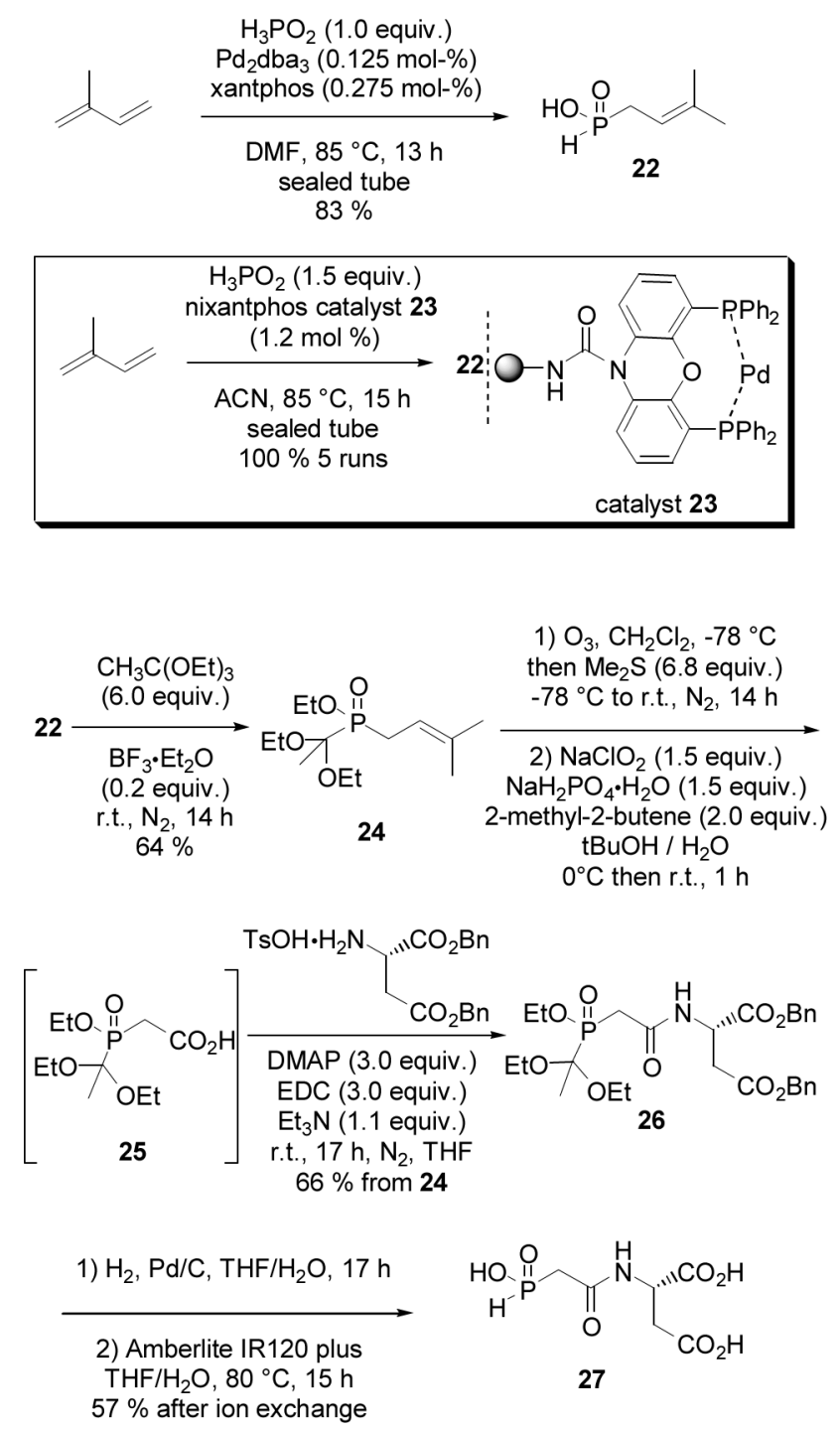

Scheme 4.

Improved synthesis of ATCase inhibitor 27. 

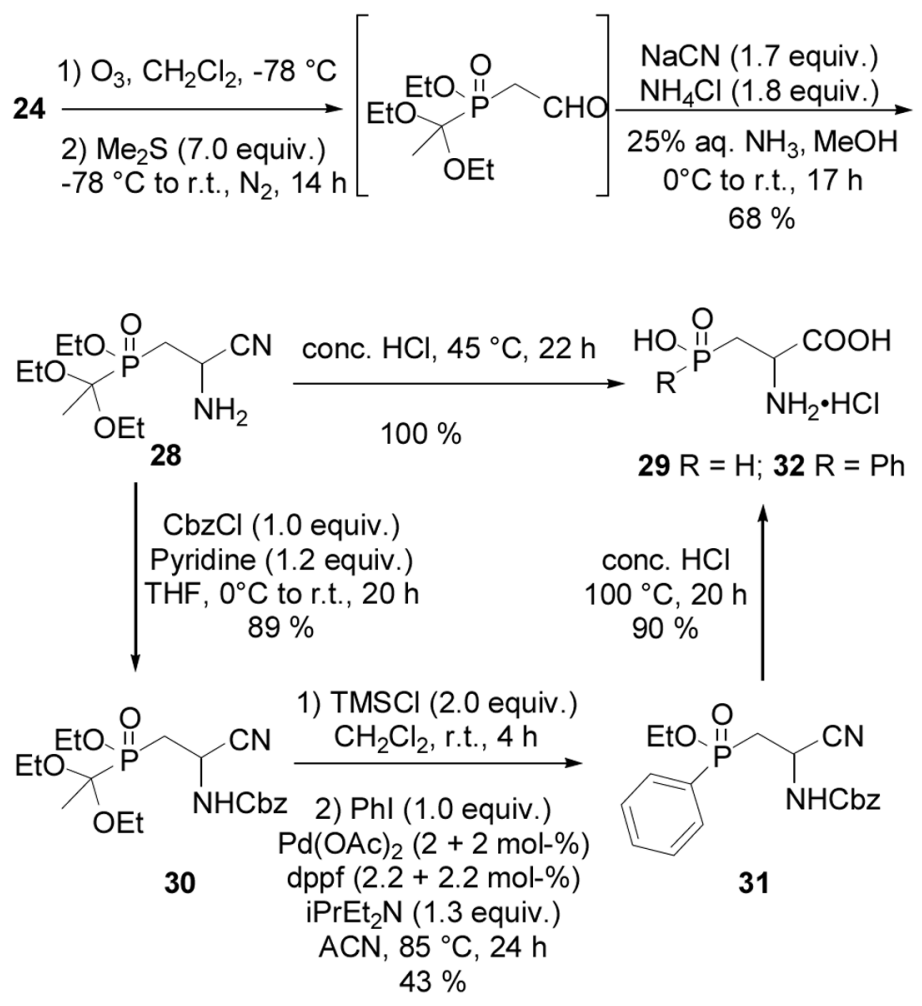

Scheme 5.

Syntheses of phosphinoaspartate analog 29 and kynureninase inhibitor 32. 


\section{Table 1}

Literature references to access protected phosphinates. ${ }^{[a]}$

\begin{tabular}{lcccc}
\hline & $\mathbf{H}_{3} \mathbf{P O}_{2}$ & $\mathbf{R}_{2} \mathbf{P C l}$ & $\mathbf{R P O}_{2} \mathbf{H}_{2}$ & $\mathbf{R P}(\mathbf{O})\left(\mathbf{O R}^{1}\right) \mathbf{H}$ \\
\hline$(\mathrm{EtO})_{3} \mathrm{CH}$ & 1 & 7 & 5 & $1 \mathrm{a}, 1 \mathrm{~b}, 8$ \\
$(\mathrm{EtO})_{3} \mathrm{CMe}$ & 1 & 7 & this work & $1 \mathrm{~g}, 9$ \\
$\mathrm{H}_{2} \mathrm{C}=\mathrm{C}(\mathrm{OEt})_{2}$ & 6 & - & - & 10 \\
$\mathrm{TIPSCl}_{2} / \mathrm{BH}_{3}$ & 4 & - & 4 & 4 \\
\hline
\end{tabular}

${ }^{[a]}$ see References. 
Table 2

Protection of $H$-phosphinates

Entry




\begin{tabular}{|c|c|c|c|}
\hline Entry & Phosphinate & Conditions $^{[a]}$ & Yield $(\%)^{[b]}$ \\
\hline \multirow[t]{2}{*}{13} & & $\mathrm{D}$ & ${ }_{58}^{[d]}$ \\
\hline & & $\mathrm{E}$ & ${ }_{74}^{[d]}$ \\
\hline
\end{tabular}

${ }^{[a]}$ Method A: $\mathrm{BF}_{3} \cdot \mathrm{Et}_{2} \mathrm{O}$ (0.2 or 0.5 equiv.), $\mathrm{CH}_{3} \mathrm{C}(\mathrm{OEt}) 3$ (6.0 equiv.), r.t., $\mathrm{N}_{2}, 16-24 \mathrm{~h}$; Method B: $\mathrm{HC}(\mathrm{OEt}) 3$ (60 equiv.), reflux, $\mathrm{N}_{2}, 24$ h; Method C: $\mathrm{Yb}(\mathrm{OTf})_{3}$ (3 mol-\%), $\mathrm{CH}_{3} \mathrm{C}(\mathrm{OEt})_{3}$ (6.0 equiv.), THF (0.4 M), r.t., $\mathrm{N}_{2}, 22 \mathrm{~h}$; Method D: $\mathrm{BF}_{3}{ }^{\bullet} \mathrm{Et}_{2} \mathrm{O}$ (1.0 equiv.), $\mathrm{CH}_{3} \mathrm{C}(\mathrm{OEt})_{3}(6.0$ equiv.), r.t., $\mathrm{N}_{2}, 4 \mathrm{~h}$ then at $0^{\circ} \mathrm{C}$, pyridine (10.0 equiv.) and $\mathrm{Ac}_{2} \mathrm{O}$ (5.0 equiv.), r.t., $\mathrm{N}_{2}, 4$ h; Method $\mathrm{E}: \mathrm{Yb}(\mathrm{OTf}) 3(3 \mathrm{~mol}-\%)$, $\mathrm{CH}_{3} \mathrm{C}(\mathrm{OEt}) 3(6.0$ equiv.), r.t., $\mathrm{N}_{2}, 3 \mathrm{~h}$ then at $0^{\circ} \mathrm{C}$, pyridine (10.0 equiv.) and $\mathrm{Ac}_{2} \mathrm{O}$ (5.0 equiv.), r.t., $\mathrm{N}_{2}, 4 \mathrm{~h}$.

${ }^{[b]}$ Isolated yields (unless otherwise noted).

${ }^{[c]}$ NMR yield.

${ }^{[d]}$ Acetate. 